\title{
1 LORE homomerization is required for 3-OH-C10:0 induced immune signaling
}

2 Sabine Eschrig ${ }^{1}$, Milena Schäffer ${ }^{1}$, Tina Illig ${ }^{1}$, Sonja Eibel ${ }^{1,3}$, Lin-Jie Shu ${ }^{1}$, Atiara Fernandez ${ }^{1,2}$,

3 Stefanie Ranf ${ }^{*}$

$4{ }^{1}$ Phytopathology, TUM School of Life Sciences, Technical University of Munich, Freising-

5 Weihenstephan, Germany

$6 \quad{ }^{2}$ Current address: Center for Plant Molecular Biology (ZMBP), University of Tübingen, Tübingen,

7 Germany

$8{ }^{3}$ Current address: Bavarian State Research Center for Agriculture, Institute for Crop Science and

9 Plant Breeding, Freising, Germany

${ }^{*}$ Correspondence: ranf@wzw.tum.de

\section{ABSTRACT}

Perception and processing of various internal and external signals is essential for all living organisms. Plants have an expanded and diversified repertoire of cell surface-localized receptorlike kinases (RLKs) that transduce signals across the plasma membrane. RLKs often assemble into higher-order receptor complexes with co-receptors, regulators and scaffolds to convert extracellular stimuli into cellular responses. To date, the only S-domain-RLK from Arabidopsis thaliana with a known ligand and function is AtLORE, a pattern recognition receptor that senses bacterial 3-hydroxy fatty acids of medium chain length, such as 3-hydroxy decanoic acid (3-OHC10:0), to activate pattern-triggered immunity. Here we show that AtLORE forms receptor homomers, which is essential for 3-OH-C10:0-induced immune signaling. AtLORE homomerization is mediated by the transmembrane and extracellular domain. We show natural variation in the perception of 3-OH-C10:0 within the Brassicaceae family. Arabidopsis lyrata and Arabidopsis halleri do not respond to $3-\mathrm{OH}-\mathrm{C} 10: 0$, although they possess a putative LORE orthologue. We found that LORE orthologues of these 3-OH-C10:0 nonresponsive species have defective extracellular domains that can bind the $3-\mathrm{OH}-\mathrm{C} 10: 0$ ligand but lack the ability to homomerize. Our findings shed light on the activation mechanisms of AtLORE and explain natural variation of 3-OH-C10:0 perception within the Brassicaceae family.

KEYWORDS

Receptor-like kinase, S-domain, LORE, homomerization, pattern recognition receptor, pattern- 


\section{INTRODUCTION}

Perception, processing and integration of a wide range of environmental and cellular stimuli is fundamental to all living organisms. In plants, this is implemented, amongst others, by members of the super-families of receptor-like kinases (RLKs) and receptor-like proteins (RLPs) (Shiu and Bleecker, 2001, 2003; Hohmann et al., 2017; Jamieson et al., 2018; Dievart et al., 2020). They regulate various cellular processes such as growth, development, reproduction or immunity (Shiu and Bleecker, 2001; De Smet et al., 2009; Li and Yang, 2016; Boutrot and Zipfel, 2017; Gou and $\mathrm{Li}, 2020$ ). Most RLKs comprise an extracellular, presumably ligand binding domain (ECD) with a variety of sequence motifs, a single-span transmembrane domain (TMD) and an intracellular domain (ICD) comprising a serine/threonine protein kinase domain for signal transduction (Shiu and Bleecker, 2001; Dievart et al., 2020). In many cases, additional tyrosine phosphorylation activity was observed. Presumably, RLKs are generally dual-specificity kinases, (Bojar et al., 2014; Macho et al., 2015), but pseudo kinases also exist. RLPs, lacking an intracellular kinase domain, are incapable of signal transduction on their own and require signaling competent partners (Jamieson et al., 2018). RLKs and RLPs are classified according to their ECD motifs, such as leucine-rich-repeat (LRR), L-type lectin (Lec), S-domain (SD) or lysin-motif (LysM) domains (Shiu and Bleecker, 2001, 2003; Dievart et al., 2020).

A common theme of RLK and RLP signaling is their association into higher order receptor complexes via homo- or hetero-oligomerization. They can act as ligand-binding receptors, coreceptors, scaffolds or positive/negative regulators to orchestrate and fine tune signaling (Ma et al., 2016b; Burkart and Stahl, 2017; Wan et al., 2019; Gou and Li, 2020). To date, we have gained a detailed molecular and structural understanding of ligand binding, activation mechanisms and the role of receptor complex formation for a few RLKs, which have become important receptor models. One prototypical heteromeric signaling complex is the heterodimer between LRR-RLK FLAGELLIN SENSING 2 (AtFLS2) and its LRR-co-receptors of the SOMATIC EMBRYOGENESIS RECEPTOR-LIKE KINASE (AtSERK) family, particularly BRI1-ASSOCIATED RECEPTOR KINASE 1/SERK3 (AtBAK1) from A. thaliana (Gomez-Gomez and Boller, 2000; Chinchilla et al., 2006; Chinchilla et al., 2007; Roux et al., 2011). FLS2 functions as pattern recognition receptor (PRR) in plant immunity. PRRs perceive microbe-associated molecular patterns (MAMPs) to induce pattern-triggered immunity (PTI) (Boutrot and Zipfel, 2017). Perception of the ligand, the peptide epitope flg22 of bacterial flagellin, triggers association of the AtFLS2 receptor with its coreceptors (Boller and Felix, 2009; Robatzek and Wirthmueller, 2013; Couto and Zipfel, 2016). Cocrystallization of the ECDs of both AtFLS2 and AtBAK1 with flg22 shows a series of intermolecular bonds that stabilize the heterodimer in both ligand- and receptor mediated manner (Sun et al., 2013b). State of the art imaging techniques enable real-time analysis of receptor dynamics in vivo and suggested that AtFLS2/AtBAK1 heterodimers can further associate into tetrameric complexes (Somssich et al., 2015). The multicomponent PRR complex for perception of the fungal MAMP chitin is characterized in detail as well. In Arabidopsis, three LysM-RLKs (LYK), AtLYK5, AtLYK4 and the CHITIN ELICITOR RECEPTOR KINASE 1 (AtCERK1/ AtLYK1) cooperate in chitin perception and signaling (Miya et al., 2007; Cao et al., 2014; Xue et al., 2019; Gong et al., 2020). Upon chitin binding, the pseudo-kinase AtLYK5 hetero-dimerizes with AtCERK1, triggering further homomerization of $A$ tCERK1. This leads to the formation of a sandwich-like receptor complex to enable downstream signaling (Couto and Zipfel, 2016; Gong et al., 2020). AtLYK4 interacts with both $A t L Y K 5$ and $A t C E R K 1$ and was reported to have a role in scaffolding or ligand binding (Gong et al., 2020). 
While ligand induced hetero-dimerization with co-receptors is essential for signaling in the previous examples, the mode of action is different for S-LOCUS RECEPTOR KINASES (SRKs) from Brassica rapa. BraSRKs belong to the family of S-domain-RLKs (SD-RLKs, also referred to as G- or B-type lectin RLKs) which comprises about 40 members in Arabidopsis and more than 100 in rice (Shiu and Bleecker, 2001; Shiu et al., 2004; Vaid et al., 2012; Xing et al., 2013; Teixeira et al., 2018). SRKs and their ligands, namely S-LOCUS CYSTEIN-RICH PEPTIDE (SCRs), mediate self-incompatibility (SI) to maintain genetic variability by avoiding inbreeding (Ivanov et al., 2010; Nasrallah and Nasrallah, 2014; Jany et al., 2019). Recognition of pollen-secreted SCRs by SRKs expressed in the stigma enables the plant to detect and subsequently reject self-pollen (Nasrallah and Nasrallah, 2014; Jany et al., 2019). The ECD of SRKs comprises two G-type lectinlike, an epidermal-growth factor (EGF)-like and a plasminogen-apple-nematode (PAN) domain, representing one typical domain architecture of SD-RLKs (Shiu and Bleecker, 2001; Xing et al., 2013; Dievart et al., 2020). Spontaneous and ligand-independent homomerization of SRKs via the ECD has been reported (Giranton et al., 2000; Naithani et al., 2007). However, the crystal structures obtained from BraSRK9 and engineered BraSRK8 elucidated, that two SKRs and two of the respective SCR peptides form a 2:2 hetero-tetrameric complex in a ligand- and receptormediated manner (Ma et al., 2016a; Murase et al., 2020). Receptor homo-dimerization of SRKs appears to be essential for SRK signaling. To date, no co-receptors are found to be involved in SRK receptor complexes.

Apart from SRKs, only a few SD-RLKs from different plant species have been described in more detail. They are involved in plant-pathogen interactions, symbiosis or abiotic stress responses (Navarro-Gochicoa et al., 2003; Chen et al., 2006; Kanzaki et al., 2008; Kim et al., 2009b; Kim et al., 2009a; Gilardoni et al., 2011; Chen et al., 2013; Cheng et al., 2013; Sun et al., 2013a; Zou et al., 2015; Fan et al., 2018; Schnepf et al., 2018; Labbé et al., 2019; Jinjun et al., 2020; Pan et al., 2020; Sun et al., 2020; Liu et al., 2021; Zhou et al., 2021). In Glycine soja, S-LOCUS LecRK (GsSRK) regulates osmotic homeostasis and plant architecture under salt stress (Sun et al., 2013a; Sun et al., 2018). Oryza sativa OsPi-d2 confers resistance to rice blast caused by the fungus Magnaporthe grisea (Chen et al., 2006). Interestingly, natural variation in a single amino acid position in the TMD appears to define resistant or susceptible alleles (Chen et al., 2006; Li et al., 2015). Expression of the LARGE SPIKE S-DOMAIN RECEPTOR LIKE KINASE 1 (OsLSK1) in rice is regulated by growth hormones and is associated with abiotic stress sensitivity and yield. The ECD of OsLSK1 interacts with itself and heteromerizes with the ECDs of five homologous SD-RLKs. Overexpression of truncated OSLSK1 lacking the ICD increases plant height and grain yield, supposedly by exerting a dominant negative effect on endogenous OsLSK1 signaling (Zou et al., 2015). Although a growing number of members of the large SD-RLK sub-family are being characterized, their ligands, receptor complex formation and downstream signaling mechanisms remain largely unknown. Biochemical characterization of SD-RLKs and identification of ligands is proving to be particularly challenging. Large-scale expression and purification of SD-RLKs appears to be especially difficult, likely because of their complex folding, high degree of glycosylation, and tendency of protein aggregation (Murase et al., 2020; Sun et al., 2020).

In $A$. thaliana, we previously discovered the SD-RLK AtLORE (LIPOOLIGOSACCHARIDE SPECIFIC REDUCED ELICITATION or SD1-29) as PRR for bacterial medium-chain 3hydroxylated fatty acid (3-OH-FA) metabolites (Ranf et al., 2015; Kutschera et al., 2019). 3-OHFAs are sensed in a chain-length and hydroxylation specific manner (Kutschera et al., 2019). Only 3-OH-FAs with acyl chains comprising 8 - 12 carbon atoms activate AtLORE signaling, with 3hydroxydecanoic acid (3-OH-C10:0) being the strongest elicitor in $A$. thaliana. 3-OH-C10:0 directly binds to the AtLORE ECD, as evidenced by microscale thermophoresis (MST) and ligand 
depletion-binding assays (Kutschera et al., 2019; Shu et al., 2021). AtLORE is the first SD-RLK in A. thaliana with a known ligand and thus a controllable means of activation, making it an important model for mechanistic studies of the SD-RLK family. Recently, downstream signaling components of AtLORE were elucidated. Sensing of 3-OH-C10:0 leads to phosphorylation of AtLORE at Y600, which is required for phosphorylation and activation of the receptor-like cytoplasmic kinase PBS1LIKE 34 (AtPBL34). AtLORE directly interacts with AtPBL34 and its homologs AtPBL35 and AtPBL36, which seem to redundantly facilitate downstream signaling in AtLORE-mediated immune responses (Luo et al., 2020). To date, no co-receptor has been found to be involved in AtLORE signaling and the steps of receptor complex formation are unknown.

Here, we show that AtLORE forms receptor homomers via TMD and ECD, which is required for 3-OH-C10:0-induced immune signaling. Interestingly, we found natural variations in responsiveness to $3-\mathrm{OH}-\mathrm{C} 10: 0$ in some closely related Brassicaceae, which have LORE orthologues with high protein sequence identity to AtLORE. Functional analysis of these orthologues and chimera with AtLORE shows that orthologues from nonresponsive species have defects in their ECDs. Interestingly, the defective LORE orthologues still bind the 3-OH-C10:0 ligand via their ECDs but are compromised in homomerization. This highlights that receptor homomerization is essential for LORE-dependent immune signaling. Moreover, our results indicate that in the case of LORE, ligand binding is independent of homomerization, in contrast to ligand- and receptor-mediated homodimerization of BraSRKs. Our results show that two Brassicaceae species closely related to $A$. thaliana lack the ability to respond to $3-\mathrm{OH}-\mathrm{C} 10: 0$, which is explained by altered homomerization ability. This sheds light on the mechanistic aspects of 3-OH-C10:0 sensing in Brassicaceae.

\section{RESULTS}

\section{AtLORE forms receptor homomers in planta}

We performed multiple sequence alignments (MAFFT algorithm, Jalview) (Fig. S1) of the amino acid sequence of the ECDs of AtLORE (AT1G61380) and well-studied SRKs from Brassica rapa, BraSRK9 (BAA21132.1) and BraSRK8 (BAF91375). Labeling of percentage identity shows a high sequence similarity and conservation of disulfide bridge forming cysteine residues in the EGF and PAN domain, indicating an overall similar domain architecture of AtLORE and BraSRKs. Transient agrobacterium-mediated overexpression of kinase-active AtLORE in Nicotiana benthamiana, which lacks a putative LORE orthologue (Ranf et al., 2015), results in a cell death-like necrotic phenotype. These cell death symptoms can be visualized by trypan blue staining (Fig. S2E) and an increased chlorophyll fluorescence (Fig. 1A), which is associated with cell death (Landeo Villanueva et al., 2021). This phenomenon is not observed upon expression of AtLORE-Km, which is mutated at the conserved ATP-binding site (K516A) in the kinase domain and has been shown to be signaling incompetent (Ranf et al., 2015). Therefore, we assume that cell death is caused by spontaneous receptor activation. Based on the high protein sequence similarity of AtLORE to BraSRKs and the receptor auto-activation of AtLORE upon transient overexpression in $N$. benthamiana, we hypothesized that AtLORE may form homomers like BraSRKs. Indeed, AtLORE homomerizes in vivo upon transient expression in $N$. benthamiana. GFP and mCherry fusion proteins of kinase-active and kinase-mutated (Km, K516A) AtLORE co-immunoprecipitate (Co-IP) with each other (Fig. 1B). AtLORE homomerization was also observed in bimolecular fluorescence complementation (BiFC) assays using AtLORE-split-YFP N-/C-terminal fragment expression (SPYCE/SPYNE) fusion proteins. To avoid background auto-fluorescence caused by 
the cell-death symptoms, AtLORE-Km was used for fluorescence-based assays. Transient coexpression of AtLORE-Km fused to either SPYCE or SPYNE restores YFP fluorescence, detectable via laser scanning microscopy and fluorescence quantification (Fig. S2A-C). We performed Förster resonance energy transfer (FRET) fluorescence lifetime imaging (FLIM) to proof direct physical LORE-LORE interaction (Fig. 1C and Fig. S2D). Co-expression of AtLORE$\mathrm{Km}$-GFP and AtLORE-Km-mCherry results in a significant reduction in GFP fluorescence lifetime T compared to the FRET-donor only control AtLORE-Km-GFP. mCherry-tagged Glutathione-S transferase of $A$. thaliana (AT1G17170, AtGST-mCherry), was used as co-expression control and does not reduce GFP fluorescence lifetimes. An AtLORE-Km-GFP-mCherry fusion protein serves as FRET positive control. AtLORE homomerization is not influenced by the presence of its ligand 3-OH-C10:0 in FRET-FLIM experiments (Fig. 1D). Thus, under the experimental conditions used, AtLORE forms homomers in the plasma membrane in a ligand-independent manner.

\section{AtLORE homomerization is mediated by the extracellular- and transmembrane domain}

To identify the region of AtLORE that mediates its homomerization, we generated truncated variants of AtLORE containing different combinations of ICD, ECD or TMD (Fig. 2A). Full length AtLORE-Km-HA co-immunoprecipitates with full length AtLORE-Km-GFP, AtLORE-ECD-TMDGFP and weakly with AtLORE-TMD-ICD-Km-GFP upon transient expression in $N$. benthamiana leaves (Fig. 2B). No co-immunoprecipitation is detected with apoplastic AtLORE-ECD-GFP, cytosolic AtLORE-ICD-Km-GFP or cytosolic GFP. This indicates that ECD and TMD contribute to LORE homomerization. In FRET-FLIM experiments, both AtLORE-ECD-TMD-mCherry and AtLORE-TMD-ICD-Km-mCherry significantly reduce the GFP fluorescence lifetime of the FRET donor AtLORE-Km-GFP (Fig. 2C and S3). Furthermore, AtLORE-ECD-TMD lacking the ICD can still homomerize and interact with AtLORE-TMD-ICD (Fig. 2D). In the latter case, only the TMDs can serve as an interaction interface, yet GFP fluorescence lifetime is reduced, highlighting the contribution of both ECD and TMD to AtLORE homomerization.

\section{A. Iyrata and A. halleri have LORE orthologues, but do not respond to 3-OH-C10:0}

Phylogenetic analysis shows that LORE is restricted to the plant family of Brassicaceae (Ranf et al., 2015). Putative LORE orthologues are encoded in the genomes of Arabidopsis halleri (AhalORE, Araha.6790s0007.1), Arabidopsis lyrata (AlyrLORE, AL2G04470) and Capsella rubella (CrubLORE, CARUB_v10021901mg) (Fig. S1). Therefore, we analyzed these species for the production of reactive oxygen species (ROS) (Fig. 3A), which is a typical PTI response to application of 3-OH-C10:0 in A.thaliana (Kutschera et al., 2019). Interestingly, we found that, in contrast to $C$. rubella and $A$. thaliana, $A$. lyrata and $A$. halleri do not respond to 3-OH-C10:0 elicitation. All four species produced ROS upon flg22 treatment (Fig. S4B). This suggests that functional PTI signaling pathways (Yu et al., 2017) and components for ROS production are in principle functional. That $A$. lyrata and $A$. halleri do not respond to 3-OH-C10:0 can originate from diverse failures, such as lack of expression, morphological differences that hinder the access of 3-OH-C10:0 to the PRR or a dysfunctional LORE protein. For further analysis, we extracted RNA from untreated leaf material of $C$. rubella, $A$. lyrata and $A$. halleri and transcribed it into cDNA to clone the coding sequence (CDS) of LORE. We obtained full length CDS clones of all orthologues, confirming that $L O R E$ is expressed in $A$. lyrata and $A$. halleri leaf tissue used for the ROS assay (Fig. 3A). Amino acid sequences of AlyrLORE and AhaLORE slightly varied from publicly available database sequences (Fig. S8). Furthermore, transient expression of GFP-fusions of 
LORE orthologues in $N$ benthamiana shows that they localize to the plasma membrane, AhalLORE-GFP was partially mislocalized (Fig. S4A). Solanaceous $N$. benthamiana has no putative LORE orthologue and is therefore insensitive to 3-OH-C10:0 but can gain the function of 3-OH-C10:0 sensing by transient expression of AtLORE (Ranf et al., 2015; Kutschera et al., 2019). Therefore, we tested the cloned LORE orthologues for functionality in gain-of-function (GOF) ROS assays in $N$. benthamiana (Fig. 3B). Expression of AtLORE or CrubLORE, but not AhalLORE or AlyrLORE resulted in a ROS response to treatment with 3-OH-C10:0. Taken together, these data support that the LORE orthologues of $A$. lyrata and $A$. halleri are the underlying factor in the inability of these species to respond to $3-\mathrm{OH}-\mathrm{C} 10: 0$.

\section{LORE orthologues have elicitor binding capacity}

To identify the region of AlyrLORE that causes its dysfunction, we created reciprocal domain swaps (DS) between ICD and ECD of AtLORE and AlyrLORE and tested those domain swaps (DS) for their GOF-ROS responses in N. benthamiana (Fig. 4A). Interestingly, we could render AtLORE inactive by replacing the ECD and TMD with AlyrLORE-ECD-TMD. In contrast, AtLORE remains functional, when the AtLORE-ICD is substituted by AlyrLORE-ICD. From this we can conclude that $A$ lyrLORE has a signaling-competent kinase domain and a dysfunctional ECD. Next, we tested whether the AlyrLORE-ECD is compromised in ligand binding. We have previously shown with ligand-depletion assays that AtLORE and CrubLORE, but not closely related AtSD123 (AT1G61390) can bind 3-OH-C10:0 (Shu et al., 2021). Here, we additionally tested the 3-OHC10:0 binding capacities of the ECDs of AlyrLORE and AhalORE expressed and harvested from $N$. benthamiana apoplasts in ligand-depletion assays. Equal amounts of total protein (Fig. S5) were incubated with $3-\mathrm{OH}-\mathrm{C} 10: 0$ and mixtures were filtered through membranes with a $30 \mathrm{kDa}$ molecular weight cut-off. The presence of unbound 3-OH-C10:0 in the filtrate was tested in a bioassay by measuring the cytosolic calcium response of AtLORE-overexpressing (OE) $A$. thaliana seedlings. When the ECDs bind the elicitor, the filtrates do not contain 3-OH-C10:0 and do not elicit a calcium response. Interestingly, the ECDs of all tested LORE orthologues bind 3$\mathrm{OH}-\mathrm{C} 10: 0$ and completely deplete $3-\mathrm{OH}-\mathrm{C} 10: 0$ from the filtrates, as no calcium responses is detectable after filtrate application (Fig. 4B). Since neither the kinase activity nor the ligand binding of AlyrLORE appears to be affected, the impaired 3-OH-C10:0 perception of AlyrLORE must have other causes.

\section{LORE orthologues from 3-OH-C10:0 nonresponsive species cannot homomerize}

Interestingly, unlike overexpression of AtLORE or CrubLORE, overexpression of AlyrLORE or AhalLORE in $N$. benthamiana does not cause cell death, as determined by chlorophyll fluorescence measurements (Fig. 5A). This indicates that receptor activation, relevant for the autoimmunity phenotype, is affected in these species. Therefore, we hypothesized that AlyrLORE and AhaLORE may fail to homomerize and analyzed their ability to homomerize using FRETFLIM experiments in N. benthamiana (Fig. 5B-D). Indeed, AlyrLORE and AhahORE are unable to form homomers in vivo, whereas signaling-competent CrubLORE homomerizes similarly to AtLORE. Thus, we conclude that homomerization of LORE is crucial for the activation of signal transduction. AlyrLORE and AhaLORE can bind 3-OH-C10:0 but do not homomerize. Thus, ligand binding is independent of receptor homomerization and not sufficient to activate LOREdependent signaling. Furthermore, our data indicate that the cell death phenotype in $N$. 
benthamiana can be used as indicator of LORE receptor functionality and requires both, an active kinase domain and receptor homomerization.

\section{Mapping of homomerization region using AtLORE and AlyrLORE chimera}

Our data suggest that loss of the homomerization ability of LORE renders two Arabidopsis species, $A$. lyrata and $A$. halleri, insensitive to 3-OH-C10:0. While AlyrLORE and AhalLORE share a very high overall amino acid identity with AtLORE, they have several single amino acid polymorphisms (SAPs) in their ECDs and TMD compared to the AtLORE-ECD, distributed across all domains (Fig. S1). To narrow down the region of the ECD that causes the loss of AlyrLORE homomerization, we generated chimera between AtLORE and AlyrLORE ECDs and TMDs. We either exchanged individual domains, such as lectin 1, lectin 2, EGF or PAN domain (L1, L2, E, P, Fig. S7A) or combinations of domains (LL, EP, Fig. 6A). After verifying the expression and correct localization in $N$. benthamiana epidermal cells (Fig. S6), we analyzed homomerization of these chimera in FRET-FLIM experiments (Fig. 6 and S7B). Chimera with individual domain swaps all retained their ability to homomerize (Fig. S7B). This indicates that loss of homomerization in AlyrLORE is not caused by a single SAP or diversification of a single domain. Therefore, we tested domain combinations, namely LL, EP and full ECD-TMD swaps. Interestingly, substitution of the whole ECD of AtLORE by AlyrLORE, but not partial ECD swaps (LL, EP) impair homomerization in FRET-FLIM experiments (Fig. 6B). A similar outcome was observed, when we tested these chimeras in 3-OH-C10:0-triggered ROS or chlorophyll fluorescence assays upon overexpression in $N$. benthamiana (Fig. 4A, 6C and D). Chimera with partial ECD swaps remain signaling competent, while those with ECD-TMD exchange do not. Hence, we could not pin down a specific region of the AlyrLORE-ECD or TMD that causes the loss of homomerization. This might indicate a large, ectodomain-spanning interaction interface of $A$ tLORE.

\section{Homomerization is essential for AtLORE downstream signaling}

Collectively, our data suggests that LORE homomerization is essential for its activation and downstream signaling. To confirm this, we performed competition experiments with the truncated AtLORE-ECD-TMD variant. We have shown that AtLORE-ECD-TMD lacking the ICD can form hetero-complexes with full length AtLORE (Fig. 2B and C). The hetero-complexes are presumably signaling incompetent as they contain only one kinase domain. To outcompete AtLORE homocomplexes, AtLORE-ECD-TMD must be present in excess relative to AtLORE. We exploited the fact, that upon transient expression in N. benthamiana AtLORE-ECD-TMD accumulates to higher protein levels than full length AtLORE (Fig. 1B). To enhance the effect, we increased the ratio of Agrobacteria carrying the competitor expression plasmid (AtLORE-ECD-TMD) to those with full length AtLORE to 5:1. Indeed, AtLORE-ECD-TMD outcompetes signaling-competent full length AtLORE homomers in competition assays, as shown by impaired ROS production in $N$. benthamiana (Fig. 7). This is not observed when cytosolic mCherry is co-expressed in excess. AtLORE-ECD-TMD thus exerts a dominant-negative effect on the ROS response upon elicitation with 3-OH-C10:0. Hence, homomerization of kinase-active LORE is required to activate signaling 301 upon 3-OH-C10:0 elicitation. 


\section{DISCUSSION}

AtLORE is the first SD-RLK from $A$. thaliana with a known ligand and characterized function (Ranf et al., 2015; Kutschera et al., 2019), which makes it an important model for studying signaling mechanisms of the SD-RLK family. However, the mechanism of AtLORE receptor activation remains largely unknown. Here we show that AtLORE forms receptor homomers in vivo and that homomerization is required to activate immune signaling. In plants, numerous examples of receptor-coreceptor hetero-dimerization have been found, making it a predominant concept of receptor activation mechanisms (Burkart and Stahl, 2017; Wan et al., 2019; Gou and Li, 2020). In contrast, the receptor activation by homo-dimerization, as shown here for AtLORE, seems to be common among representatives of the SD-RLK family. The SD-RLKs OSLSK1 homomerizes and heteromerizes with five close homologs in yeast and in planta (Zou et al., 2015). Since its ligand and physiological function are unknown, the mechanistic relevance of the observed interactions also remains unclear. Homo-dimerization of BraSRKs has been studied in detail and was shown to be required for ligand binding and signal transduction (Giranton et al., 2000; Naithani et al., 2007; Shimosato et al., 2007; Ma et al., 2016a; Murase et al., 2020). To date, no co-receptors of any SD-RLK has been identified, which underlines the relevance of homo-dimerization for receptor activation. Protein homomerization analysis is particularly challenging due to uncontrollable homodimer stoichiometry. Most protein-protein interaction methods require two different protein epitope tags, whether for FRET, Co-IP, or BiFC (Xing et al., 2016). However, the combinations of protein-tags in a homomer/dimer is subject to random distribution. Statistically, only one-third of dimers have a combination of two different tags when expressed equimolar. Therefore, large proportions of homomers remain unconsidered or even influence the readout of the experiment. For example, in Co-IPs homodimers with identical tags compete with homodimers containing different tags for binding to the antibody trap, but only the latter are detectable on the immunoblot. In case of FLIM the stoichiometry of homo-dimerization results in formation of FRET-incapable (GFP-GFP/mCherry-mCherry) or FRET-capable (GFP-mCherry) pairs. The method does not provide the resolution to distinguish individual molecules. Rather, the measured average GFP lifetime of each pixel is the result of a random mixture of all possible dimers. This might explain, why the GFP lifetime is only moderately reduced for AtLORE homomers compared to the FRETpositive control, as FRET-incapable AtLORE-GFP homodimers diminish the effect of the FRETcapable AtLORE-GFP-AtLORE-mCherry dimers.

BraSRKs are thought to pre-assemble into homo-dimers in a ligand-independent manner and these preformed complexes allow ligand binding and a rapid activation of the receptor (Giranton et al., 2000; Naithani et al., 2007; Shimosato et al., 2007). Ligand binding might enhance BraSRK dimerization by rearrangement of these pre-assembled complexes (Shimosato et al., 2007). However, whether preformed receptor complexes exist prior to ligand binding under physiological conditions is controversially discussed. AtCERK1 forms homo-dimers independent of its ligand chitin upon overexpression in $A$. thaliana (Liu et al., 2012), whereas under physiological conditions AtCERK1 homo-dimerization was fully dependent on AtLYK5 and chitin binding (Cao et al., 2014; Gong et al., 2020). Ligand-independent self-association of AtFLS2 was also reported (Sun et al., 2012). FLIM analysis of AtFLS2 at the membrane did not show homomerization, leading to the speculation that AtFLS2 might only self-associate upon internalization in a ligand-independent manner (Somssich et al., 2015). Our FRET-FLIM data show that AtLORE homomerization is ligand-independent, at least under the experimental conditions used. We could not detect any significant differences in the homomerization state of AtLORE between 10-20 minutes after application of 3-OH-C10:0. Although receptor complex formation should be a rapid process, this timeframe was suggested for comparable FLIM studies (Somssich et al., 2015). The authors 
discussed that a relatively large proportion of complexes need to accumulate to change the average lifetime of one pixel in an FLIM image. To avoid strong overexpression which might lead to artifacts as shown for AtCERK1(Liu et al., 2012), we used an inducible promotor system for an adjustable and more moderate protein expression. To date we can only speculate how AtLORE homomerization acts under physiological conditions as protein expression driven by the endogenous LORE-promotor is below the detection limit for fluorescence-based assays. At present our data do not allow us to draw conclusions about the oligomerization state of AtLORE. However, for BraSRKs oligomerization into higher order complexes was suggested by crosslinking experiments and velocity sedimentation on sucrose gradients (Giranton et al., 2000), but its physiological relevance remains unclear. Such oligomerization dynamics of receptor complex formation was also reported for other receptor complexes, such as AtFLS2-AtBAK1 (Somssich et al., 2015). The plant may maintain receptor complexes in a stable preformed steady-state to ensure rapid signal transduction that is fine-tuned by multiple regulators in higher-order complexes.

Our data suggest that homomerization of AtLORE is mediated by the ECD and TMD. In Co-IPs, neither the apoplastic ECD nor the cytosolic ICD could interact with full-length AtLORE, suggesting that membrane anchoring is required for dimerization. However, it is also possible that the interaction surface of the membrane-bound full-length AtLORE is not accessible for the soluble truncations. Interestingly, AtLORE-ECD-TMD and AtLORE-TMD-ICD can interact in FRET-FLIM experiments. In this combination, only the TMDs provide a potential interaction surface suggesting that the TMD substantially contributes to AtLORE homomerization. TMD helixes represent a typical interaction interface in the hydrophobic environment of the membrane, which often contain conserved TMD interaction motifs (Herrmann et al., 2009; Langosch and Arkin, 2009; Fink et al., 2012). The relevance of TMDs for receptor complex formation is highly discussed in the animal field (Westerfield and Barrera, 2020). Involvement of TMDs in dimerization has also been shown for several plant RLKs. The TMD of the RLK SUPPRESSOR OF BRI1-1 (SOBIR1) possesses a GxxxG-motif, which mediates high affinity TMD-TMD association and is crucial for its interaction with the RLK Cf-4 (Bi et al., 2015). The TMD of the RLK Arabidopsis CRINKLY 4 (ACR4) homodimerizes (Stokes and Gururaj Rao, 2008) and is specifically required for the interaction with the RLK CLAVATA1 (CLV1) (Stahl et al., 2013). The relevance of TMDs in SD-RLK signaling is underlined by OsPi-d2, in which a single amino acid polymorphism in the TMD (I441M) determines susceptible or resistant alleles towards rice blast. For BraSRKs it was hypothesized that the TMDs may contribute to the preformed SRK dimer, which is then strengthened by ligand binding (Shimosato et al., 2007; Ma et al., 2016a). Interestingly, high-affinity binding of SCR8 peptides was only observable for membrane-bound SRK8 variants containing the ECD, TMD and parts of the juxta-membrane domain, but not for soluble ECDs (Takayama et al., 2001; Shimosato et al., 2007). An artificially dimerizing form of the SRK8-ECD, achieved by integration of a helix-loophelix zipper domain, exhibited a high SCR binding affinity, which highlights the importance of membrane anchorage for ligand binding and dimerization (Shimosato et al., 2007). BraSRK homodimerization was furthermore shown to be mediated by the ECD in yeast-two-hybrid studies, while kinase domains do not self-associate (Naithani et al., 2007). Especially the PAN and partially the EGF domains, but not the lectin domains exhibited homo-dimerization capacities in yeast (Naithani et al., 2007). For BraSRK9 and engineered BraSRK8, crystal structures of their ECDs show both ligand- and receptor mediated interaction (Ma et al., 2016a; Murase et al., 2020). In both cases, an BraSRK-ECD dimer co-crystallized with two BraSCR peptide ligands, forming a tetrameric 2:2 receptor-ligand complex. Is it suggested that BraSCRs majorly contribute to complex formation by cross-linkage of the BraSRK monomers. Although studies about BraSRKs provide a detailed insight into homo-dimerization, the comparability between AtLORE and BraSRKs is limited as 
AtLORE binds a very small chemical compound instead of a peptide ligand. So far, the binding site of 3-OH-C10:0 in the AtLORE-ECD is unknown. Due to the small size of the ligand, one must assume a relatively small interface between the receptor and the ligand, which may not be sufficient to crosslink two AtLORE monomers or to enhance homo-dimerization in a manner comparable to SRK-SCR complexes. Interestingly, we found that AlyrLORE and AhalLORE cannot homo-dimerize but bind the 3-OH-C10:0 ligand. Thus, 3-OH-C10:0 can bind to LORE monomers. One may speculate that dimerization is stabilized by conformational changes rather than ligand-mediated cross-linking. Taken together, it seems reasonable that AtLORE may form pre-assembled complexes in a ligand-independent manner. However, the underlying molecular mechanism of receptor activation remains unclear.

AtLORE homomerization is essential for receptor activation and downstream signaling. We demonstrate that LORE orthologues of 3-OH-C10:0 insensitive species are impaired in their homomerization capacity, and therefore cannot mediate downstream signaling. Truncated AtLORE containing only ECD and TMD exerts a dominant negative effect on 3-OH-C10:0 induced signaling. We assume that this effect is caused by formation of non-functional heterodimers with full-length AtLORE, which lack downstream signaling capacities. Competition for ligand binding seems unlikely given the high ligand concentrations used in our experiments. Similar competitionbased phenomena have been repeatedly described for other RLKs. Overexpression of truncated OsLSK1 lacking the kinase domain resulted in increased plant height and yield in rice (Zou et al., 2015). OsLSK1 was shown to interact with itself and five homologous proteins. Truncated OsLSK1 may interfere with signaling of OsLSK1 and the other interacting SD-RLKs or may compete for ligand binding. Upon overexpression of AtFLS2 truncations lacking the LRR domain a similar effect was observable. The truncations associate with full-length AtFLS2 and exert a dominant negative effect on flg22-induced signaling (Sun et al., 2012). Taken together, these studies show that analysis of a dominant negative effect, which is especially applicable for homomerizing RLKs, provides valuable insight into receptor complexes and their signaling mechanisms.

In vitro phosphorylation assay of microsomal membranes shows that oligomerization of membrane-bound recombinant BraSRKs is essential for auto-phosphorylation (Giranton et al., 2000) and that preformed BraSRK oligomers exhibit a basal constitutive auto-phosphorylation. We assume a similar mode of action for AtLORE. Strong overexpression of AtLORE in $N$. benthamiana results in cell death. We can show that this phenotype depends on both, an active kinase domain and dimerization ability, as it is completely absent in non-homomerizing LOREorthologues or kinase mutated AtLORE. Induced cell death is usually known as a highly effective mechanism of hypersensitive defense responses after pathogen recognition (Balint-Kurti, 2019). However, the phenomenon of cell death-like symptoms upon RLKs overexpression has been described repeatedly. Overexpression of AtCERK1 in N. benthamiana resulted in cell death in a kinase-dependent manner (Pietraszewska-Bogiel et al., 2013). It was hypothesized that overproduction of AtCERK1 induces ligand-independent homomerization, leading to autoactivation and dysregulated signal transduction. However, this AtCERK1 cell-death phenotype was not apparent upon stable expression in $A$. thaliana. Two L-type lectin RLKs (LecRKs), AtLecRK-IX.1 and AtLecRK-IX.2, exhibit cell death upon overexpression in both $A$. thaliana and $N$. benthamiana (Wang et al., 2015). The cell death correlated with transgene expression levels and was dependent on kinase activity, but not on extracellular lectin domains. Homomerization was not shown for AtLecRK-IX.1 and AtLecRK-IX.2, yet is also shown for other AtLecRKs (Guo et al., 2018). Taken together, this suggests that homomerizing RLKs are in particular prone to spontaneous auto-activation upon strong overexpression, resulting in cell death. In the case of 
444

LORE, we found that this phenotype can serve as a proxy and simple read-out for signaling active receptor complexes.

Interestingly, we found natural variation in 3-OH-C10:0 responsiveness within a subset of Brassicaceae species. $A$. Iyrata and $A$. halleri are closely related to $A$. thaliana and have LORE orthologues with a high degree of sequence identity to AtLORE. While trying to decipher the underlying defects in these two 3-OH-C10:0-insensitive species, we found that their orthologues can bind the 3-OH-C10:0 ligand but exhibit defective dimerization capacities, which render them signaling incompetent. Since we found a number of SAPs across the AlyrLORE-ECD, we investigated chimera of AtLORE and AlyrLORE to identify the causal region or SAP. However, only substitution by the entire AlyrLORE-ECD abolishes homomerization. We hypothesize that several SAPs collectively cause the loss of homomerization. From an evolutionary perspective, random single polymorphisms e.g. in the ligand binding site, seem more likely to lead to a loss-offunction mutation than a series of SAPs accumulating in the ectodomain leading to a loss of homomerization. This has been shown for example for FLS2. Flg22 binding capacities to putative FLS2 orthologues were analyzed in several $A$. thaliana accessions. The data suggested a direct correlation between the FLS2 binding capacities and the responsiveness to flg22 (Bauer et al., 2001). While our findings were rather unexpected from an evolutionary perspective, they may shed light on the evolutionary processes of diversification and neo-functionalization in the family of SD-RLKs which is so far rather poorly understood (Xing et al., 2013). This supports that natural variation provides a powerful molecular toolbox to improve our mechanistic understanding of RLK signaling.

\section{MATERIALS AND METHODS}

\section{Sequence alignments}

Multiple amino acid sequence alignments (MSA) (Fig. S1) were performed with Jalview (Version 2.11.1.4 (Waterhouse et al., 2009)). Amino acid sequences of respective proteins were retrieved from the Uniprot database (Consortium, 2020). For LORE, ECD regions excluding signal peptides (SP) were selected according to domain annotations given on Uniprot and in (Naithani et al., 2007) for LORE (see supplementary table 2). SP predictions of BraSRKs were done by SignalP-5.0 server (Almagro Armenteros et al., 2019). MSA of ECDs without SPs was calculated by the MAFFT (multiple alignment using fast Fourier transformation) algorithm (Katoh et al., 2019) with default settings with the in-built alignment tool of Jalview. Amino acid sequence alignment of AlyrLORE and AhalLORE cloned from cDNA and the respective sequences from genome database entries (Fig. S8) was performed using the in-built alignment tool of the SnapGene software (Insightful Science; available at snapgene.com).

\section{Plant material and cultivation}

N. benthamiana, A. thaliana Col-0, A. halleri (N9852, from P. Falter-Braun, Helmholtz Center Munich), A. lyrata (MN47, from M. Quint, MLU Halle) and C. rubella (N22697, from M. Quint, MLU Halle) were sown on standard potting soil mixed with vermiculite (9:1). All Brassicaceae seeds were stratified in the dark for at least $48 \mathrm{~h}$ at $4^{\circ} \mathrm{C}$ and then grown under short day conditions $(8 \mathrm{~h}$ light, $16 \mathrm{~h}$ darkness, $21^{\circ} \mathrm{C}$, and $60 \%$ relative humidity). $N$. benthamiana was grown under long day conditions ( $16 \mathrm{~h}$ light, $8 \mathrm{~h}$ darkness, $24^{\circ} \mathrm{C}$, and $60 \%$ humidity). Generation of $A$. thaliana Col- $0^{\mathrm{AEQ}}$ overexpressing AtLORE (CaMV35S:LORE, LORE-OE) was previously described (Shu et al., 
2021). For cytosolic $\mathrm{Ca}^{2+}$-measurements, seeds were surface-sterilized with chlorine gas $(4 \mathrm{~h})$ and seedlings grown in liquid medium (0.5x Murashige \& Skoog medium, including vitamins (Duchefa), 0.25\% sucrose, $1 \mathrm{mM} \mathrm{MES,} \mathrm{pH} \mathrm{5.7)} \mathrm{under} \mathrm{long} \mathrm{day} \mathrm{conditions} \mathrm{as} \mathrm{described} \mathrm{above.}$

\section{Molecular cloning}

All plasmids used in this study were cloned using Golden-Gate (GG) (Engler et al., 2009; Weber et al., 2011) or Gateway ${ }^{\mathrm{TM}}$ cloning techniques (Katzen, 2007). Cloning of AtLORE (AT1G61380), AtLORE-Km, CrubLORE (CARUB_v10021901mg), CrubLORE-ECD, AtSD1-23i-ECD (AT1G61390) and apoplastic mCherry was described previously (Ranf et al., 2015; Shu et al., 2021). AtGST (AT1G17170) was cloned from pGEX-6P-1. For cloning of AhalLORE (Araha.6790s0007.1) and AlyrLORE (AL2G04470), total RNA was extracted from leaf material of the respective species by the TRIzol RNA extraction method. RNA samples were treated with DNAse I (Thermo Fisher Scientific) and reverse-transcribed into cDNA using RevertAid reverse transcriptase, Ribolock RNase inhibitor and Oligo(dT) 18 primer, according to manufacturer's instructions (Thermo Fisher Scientific). Full length coding sequences (CDS) of respective genes were amplified with specific primers (Supplementary table 1) and ligated into a suitable GG-vector. Sequences cloned from cDNA of AhaLORE and AlyrLORE slightly varied from database entries (see full sequence alignments figure S8). Conserved ATP binding sites of LORE kinase domains (K516A, Km, primers see supplementary table 1) were modified by site-directed mutagenesis as previously described (Ranf et al., 2015) (primers supplementary table 1). For AhalLORE the first intron of AtLORE (109 bp) was amplified from genomic DNA and inserted into AhalLORE CDSs (at the $45^{\text {th }}$ codon) to circumvent problematic read through in E. colior Agrobacterium tumefaciens (primers supplementary table 1). AtLORE truncations and receptor chimera with AlyrLORE were designed according to domain annotations from Uniprot and Naithani et al. (2007) (see supplementary table 2). For cloning of truncations and chimera respective parts of the CDS were amplified by PCR with Bpil linkers from vectors with full length CDS (backbone PCR) and recircularized by GG techniques (see Supplementary table 1 and 3). For all membrane bound truncations, the AtLORE signal peptide was integrated. CDSs excluding STOP codons were combined with CaMV35S promotor and terminator, a C-terminal ten-glycine linker and respective C-terminal epitope tags (GFP, mCherry, SPYCE, SPYNE, hemagglutinin (HA)) using GG techniques. For FRET-FLIM experiments, CDSs were transferred via Gateway ${ }^{\mathrm{TM}}$ cloning (LRclonase $^{\mathrm{TM}}$, Thermo Fisher Scientific, according to manufacturer's manual) into a final Gateway ${ }^{\mathrm{TM}}$ vector containing an estradiol inducible trans-activator cassette (XVE) and C-terminal GFP, mCherry or GFP-mCherry fusion epitope tags (FLIM-vectors, previously described in (Bleckmann et al., 2009)). Final expression vectors were transformed into Agrobacteria tumefaciens for transient expression in planta.

\section{Transient protein expression in N. benthamiana}

$N$. benthamiana was transiently transformed by Agrobacterium-mediated transformation as described previously (Shu et al., 2021). Agrobacterium tumefaciens (strain GV3101 pMP90) carrying expression constructs were cultivated on LB agar with $30 \mu \mathrm{g} / \mathrm{mL}$ gentamycin, $10 \mu \mathrm{g} / \mathrm{mL}$ rifampicin, and $50 \mu \mathrm{g} / \mathrm{mL}$ kanamycin, or $100 \mu \mathrm{g} / \mathrm{mL}$ spectinomycin for Agrobacteria carrying FLIM expression constructs. $\mathrm{OD}_{600}$ of Agrobacteria was adjusted to 0.5 for general purposes or 0.025 for gain-of-function ROS measurement. Prior to infiltration, Agrobacteria carrying desired expression vectors or the silencing suppressor p19 were mixed in a 1:1 ratio. In case of co- 
533 expression, the ratio was adjusted to $1: 1: 1$. For ROS competition assays, the $O_{600}$ of Agrobacteria carrying p19 was 0.15, for AtLORE 0.025 and for AtLORE-ECD-TMD or cytosolic mCherry 0.125 (ratio p19:AtLORE:competing component 6:1:5). Expression of FLIM constructs driven by the inducible XVE trans-activator was induced by infiltration of $20 \mu \mathrm{M} \beta$-estradiol and $0.1 \%$ Tween20 into transformed leaf areas $24 \mathrm{~h}$ after infiltration of agrobacteria. Protein expression was observable 16-24 h after induction.

\section{Protein extraction}

Plant material was harvested (60-70 leaf discs of $\varnothing 4 \mathrm{~mm}$ ), frozen in liquid nitrogen and ground to fine powder (1 $\mathrm{min}$ at $30 \mathrm{~Hz}$ in a TissueLyzerll, Qiagen). Total protein was extracted by incubation with extraction buffer $(6 \mu \mathrm{L} /$ leaf disc, $150 \mathrm{mM}$ Tris- $\mathrm{HCl} \mathrm{pH} 7.5,150 \mathrm{mM} \mathrm{NaCl}, 10 \%$ glycerol, 1\% Nonidet-P40, $10 \mathrm{mM}$ EDTA, $1 \mathrm{mM} \mathrm{Na} \mathrm{MoO}_{4}, 1 \mathrm{mM} \mathrm{NaF}, 1 \mathrm{mM}$ DTT, 1\% (w/v) polyvinylpyrrolidon, $1 \%(\mathrm{v} / \mathrm{v})$ protease inhibitor cocktail P9599 (Sigma-Aldrich)) for $1 \mathrm{~h}$ at $4^{\circ} \mathrm{C}$. After centrifugation $\left(18000 \mathrm{~g}, 30 \mathrm{~min}, 4^{\circ} \mathrm{C}\right)$, the supernatant was used for verification of protein expression via immunoblot or Co-IP experiments.

\section{Co-immunoprecipitation}

For Co-IPs, protein extracts were incubated for $1-2 \mathrm{~h}$ at $4^{\circ} \mathrm{C}$ with GFP-Trap_MA (magnetic beads, ChromoTek, handling according to manufacturer's manual). Magnetic beads were washed three times with buffer (150 mM Tris-HCL pH 7.5, $150 \mathrm{mM} \mathrm{NaCl}, 0.5 \%$ Nonidet-P40) and re-suspended in $20 \mu \mathrm{L}$ 1xSDS-sample buffer (5x SDS sample buffer: $60 \mathrm{mM}$ Tris-HCL pH 6.8, 2\% SDS, $10 \%$ glycerol, $5 \% \beta$-mercaptoethanol, $0.01 \%$ bromophenolblue).

\section{Immunoblot}

Protein samples in $1 \times$ SDS sample buffer were denatured for $10 \mathrm{~min}$ at $95^{\circ} \mathrm{C}$ and separated by SDS-polyacrylamide gel electrophoresis (5\% stacking gel, $10 \%$ resolving gel, $60-100 \mathrm{~V}$, 1x Laemmli running buffer). Proteins were blotted onto a $0.2 \mu \mathrm{m}$ Protran $^{\mathrm{TM}}$ nitrocellulose membrane (GE healthcare) using $1 x$ transfer buffer $(3.03 \mathrm{~g} / \mathrm{L}$ Tris base, $14.4 \mathrm{~g} / \mathrm{L}$ glycine, $20 \%$ methanol, $0.05 \%$ SDS) and a semi dry transfer cell (Bio-Rad, $1 \mathrm{~mA} / \mathrm{cm}^{2}, 1 \mathrm{~h}$ ). Membrane was blocked with protein free blocking solution T20 (Pierce) and proteins were detected with respective antibodies diluted in blocking solution for primary antibodies or 1x TBS-T (6.06 g/L Tris base, $8.76 \mathrm{~g} / \mathrm{L} \mathrm{NaCl}, \mathrm{pH} 6.7,0.05 \%$ Tween20) for secondary antibodies (1:1000 anti-GFP 3H9 (ChromoTek) with 1:20000 anti-rat-HRP (A9542, Sigma-Aldrich); 1:500 c-Myc 9E10 (Santa Cruz) with 1:5000 anti-mouse sc-2031 (Santa Cruz); 1:2000 anti-HA-HRP 3F10 (Sigma-Aldrich)). After washing of membranes ( $3 \times 10 \mathrm{~min}$ in $1 \times$ TBS-T), chemiluminescence was detected with a CCD camera system (Fusion SL System, Vilber Lourmat $\mathrm{GmbH}$ ) upon incubation with a peroxidase substrate (SuperSignal®West Femto Maximum Sensitivity Substrate (Fig. 1A, S2D) or SuperSignalBWest Dura Extended Duration Substrate (Pierce) (Fig. 2B)). When immunoblots were analyzed with a second, different antibody, membranes were washed (1x TBS-T) after detection and incubated in stripping buffer (2\% SDS, $62.5 \mathrm{mM}$ Tris/ $\mathrm{HCl} \mathrm{pH} \mathrm{6.7,} \mathrm{100mM}$ $\beta$-mercaptoethanol) at $50^{\circ} \mathrm{C}$ for 30 minutes. Stripped membrane was blocked and immunodetected again. Membranes were stained for total protein with amido black (1 g/L amido black, $250 \mathrm{~mL} / \mathrm{L}$ isopropyl, $100 \mathrm{~mL} / \mathrm{L}$ acetic acid). 


\section{Bimolecular fluorescence complementation}

SPYCE/SPYNE epitope tags for BiFCs were previously described (Walter et al., 2004) and adapted for GG cloning. Respective interaction candidates were transiently co-expressed in $N$. benthamiana. YFP fluorescence complementation was visually assessed two days post infiltration via confocal laser scanning microscopy (Leica TCS SP5, ex 514nm, em 525 - $550 \mathrm{~nm}$, Z-stack of 22 planes). For YFP fluorescence quantification, leaf discs $(\varnothing 4 \mathrm{~mm}$ ) were floated on water in a black 96-well plate and fluorescence was measured with a plate reader (Tecan Infinite F200 PRO, excitation $485 \mathrm{~nm}$, emission $535 \mathrm{~nm}, 25$ flashes, integration time $20 \mu \mathrm{s}$, gain set to 70, $2 \times 2$ reads per well). Protein expression was validated by immunoblots.

\section{Trypan blue staining}

Leaf discs $(\varnothing 2 \mathrm{~cm})$ were harvested five days post transformation and incubated $\left(95^{\circ} \mathrm{C}, 5 \mathrm{~min}\right)$ with $3 \mathrm{~mL}$ trypan blue staining solution $(25 \%(\mathrm{v} / \mathrm{v})$ lactic acid, $25 \%(\mathrm{v} / \mathrm{v})$ phenol, $25 \%(\mathrm{v} / \mathrm{v})$ glycerol, $25 \%(\mathrm{v} / \mathrm{v}) \mathrm{H}_{2} \mathrm{O}, 25 \%(\mathrm{w} / \mathrm{v})$ trypan blue). Leafs were washed several times with destaining solution ( $250 \mathrm{~g}$ chloral hydrate in $100 \mathrm{~mL} \mathrm{H}_{2} \mathrm{O}$ ) and rinsed with water before photo-documentation.

\section{ROS measurement}

Accumulation of ROS was measured with a luminol based reporter system and a microplate reader (Tecan Infinite F200 PRO or Luminoscan Ascent 2.1, Thermo Fisher Scientific) as describe previously (Ranf et al., 2015). Leaf discs ( $\varnothing 4 \mathrm{~mm}$ ) of Brassicaceae (6-8 weeks old) or transiently transformed $N$. benthamiana (36 h post agro-infiltration) were floated on water in white 96-well plates for at least $6 \mathrm{~h}$ (for $N$. benthamiana) or overnight (for Brassicaceae). Prior to measurements, water was replaced with $100 \mu \mathrm{L}$ horseradish peroxidase-luminol solution $(2 \mu \mathrm{g} / \mathrm{mL}$ horseradish peroxidase, $10 \mu \mathrm{M} \mathrm{L}-012$ (WAKO Chemicals $\mathrm{GmbH}$ )). After addition of elicitors or respective controls (3-OH-C10:0 (Matreya LLC) in MeOH, flg22 (QRLSTGSRINSAKDDAAGLQIA in ddH ${ }_{2} \mathrm{O}$ ), luminescence was recorded in relative light units $(R L U)$ in 1 minute intervals for up to 60 minutes. Total ROS accumulation was summed up for elicitor and control treatments.

\section{Ligand depletion assay}

Ligand depletion assay was performed as previously described (Shu et al., 2021). ECDs of respective proteins were transiently expressed in $N$. benthamiana apoplasts and harvested via apoplastic washing fluids (AWF). AWFs were concentrated and incubated with 3-OH-C10:0 (9:1, $\mathrm{v} / \mathrm{v}$ ). Unbound elicitor was separated by filtration (30 kDa molecular weight cut-off). Filtrates were used for elicitation of cytosolic calcium signaling in LORE-OE reporter lines as described before (Ranf et al., 2015; Shu et al., 2021). For each time point, luminescence was normalized to total luminescence counts remaining $\left(\mathrm{L} / \mathrm{L}_{\max }\right)$ and total accumulation of calcium signals was summed up from 3 to 30 minutes. $5 \mu \mathrm{L}$ of concentrated AWF with a total protein concentration of $1.5 \mathrm{mg} / \mathrm{mL}$ was analyzed for protein expression by immunoblot. 
616 Red light emission of chlorophyll florescence can be measured for quantification of cell death 617 (Landeo Villanueva et al., 2021). Leaf discs $(\varnothing 4 \mathrm{~mm}$ ) of transiently transformed $N$. benthamiana 618 were floated on water in black 96-well plates and chlorophyll fluorescence was measured with a 619 plate reader (Tecan Infinite F200 PRO, excitation $535 \mathrm{~nm}$, emission $590 \mathrm{~nm}, 25$ flashes, 620 integration time $20 \mu \mathrm{s}, 4 \times 4$ reads per well, gain set to 80 ) as relative fluorescence units (RFU). 621 Values of all reads per well were summed up.

\section{Microscopy}

624 Confocal laser scanning microscopy was performed using either a Leica TCS SP5 (Argon laser, 625 HyD2 detectors) or an Olympus FV3000 system (diode lasers, PMT detectors). Images were 626 acquired with an image size of $512 \times 512$ pixels. In case of co-expression analysis, images were taken with a sequential scan to avoid 'bleed through'. GFP was excited at $488 \mathrm{~nm}$ and emission detected at 500-540 nm. mCherry was excited at $561 \mathrm{~nm}$ and emission detected at 570-620 nm. YFP was excited at $514 \mathrm{~nm}$ and emission detected at 525-550 nm. Images were imported and processed with OMERO (Allan et al., 2012).

\section{Fluorescence lifetime imaging (FLIM)}

GFP fluorescence lifetimes were measured via time-correlated single-photon counting (TCSPC) by an Olympus FV3000 system linked to a PicoQuant FCS/FLIM-FRET/rapidFLIM upgrade kit, based on previously described protocols (Weidtkamp-Peters and Stahl, 2017). TCSPC was performed with a $485 \mathrm{~nm}$ (LDH-D-C-485) pulsed laser, two TCSPC modules (TimeHarp 260 PICO Dual, TimeHarp 260 NANO Dual) and two photon counting PMA hybrid 40 detectors. Coexpressing cells were detected by confocal imaging via an Olympus FV3000 60x water immersion objective (UPLSAPO60XW 60x/NA 1.2/WD 0.28) and a selected measuring area was magnified (4x zoom). TCSPC was performed with a laser pulse rate of $40.00 \mathrm{MHz}$, a TCSPC resolution of $25.0 \mathrm{ps}$ and an image size of $512 \times 512$ pixels. 500-1000 photon counts per pixel were acquired for each image. FLIM was analyzed by the Symphotime64 software of PicoQuant via n-exponential reconvolution and an internally calculated instrument response function (IRF). A two-parameter fitting $(n=2)$ was performed in most cases. Only FLIM analysis with fitting coefficients $\left(x^{2}\right)$ between 1.0 and 2.0 were accepted. Intensity weighted average lifetimes $\mathrm{T}$ of membrane ROls were 
FIGURES

A

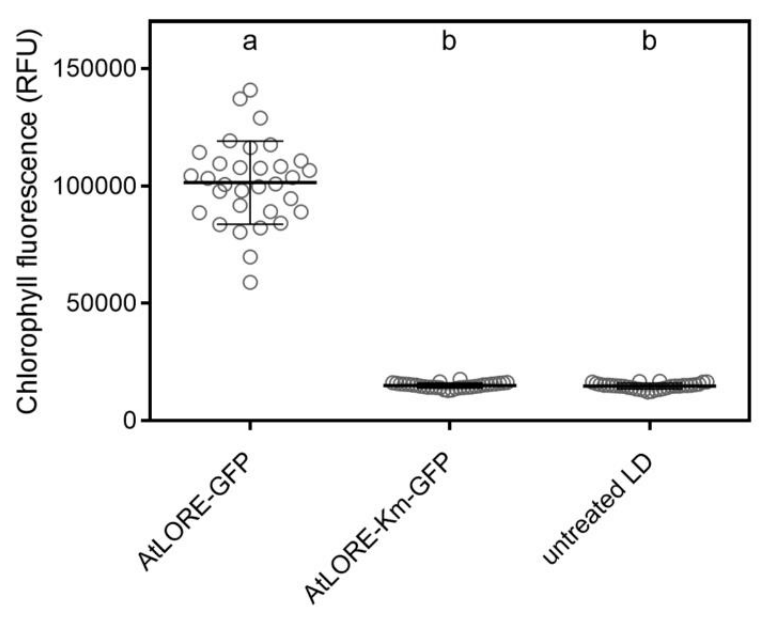

B

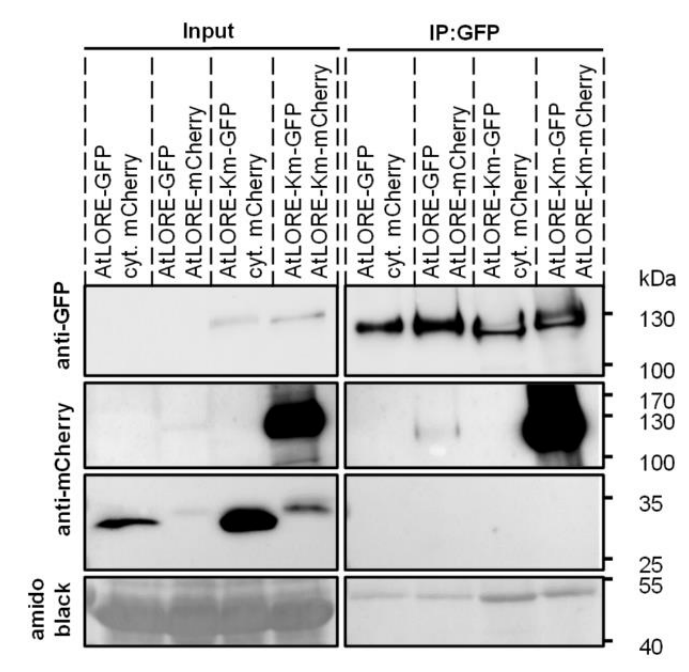

C

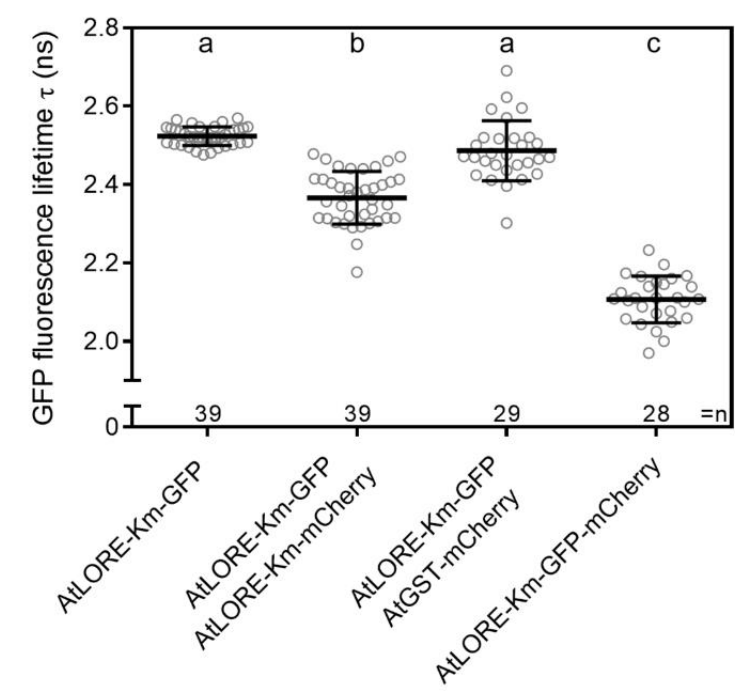

$\mathrm{D}$

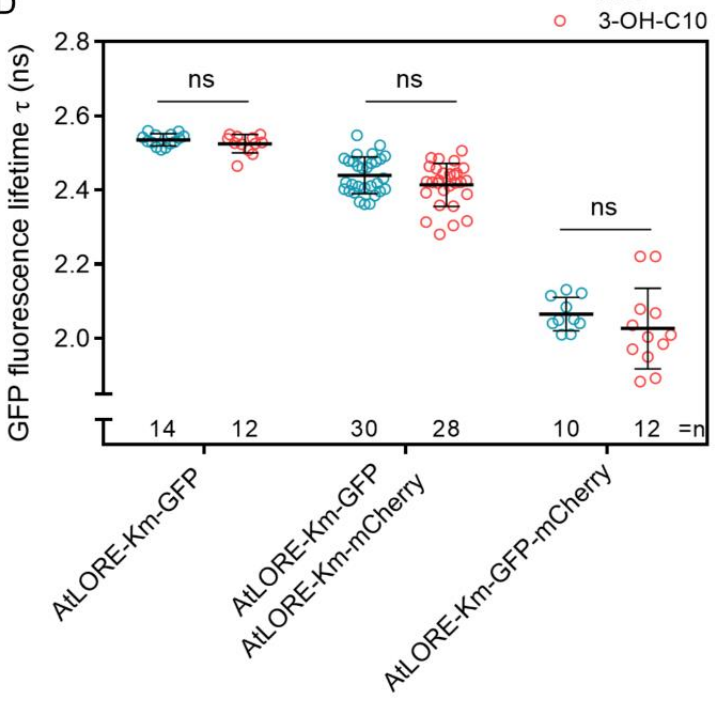

Figure 1 AtLORE forms homomers and has an auto-immunity phenotype in $N$. benthamiana. A Chlorophyll fluorescence measurements (relative fluorescence units, RFU) in $N$. benthamiana upon transient expression of candidates four days post transformation. Pooled data of two technical replicates from one biological replicate are shown. Data show mean with SD. $\mathrm{n}=32$ leaf discs. Statistics analyzed by one-way ANOVA with Tukey's multiple comparisons test, $\alpha=0.01$. Data not sharing the same letter are significantly different. B Anti-GFP and anti-mCherry immunoblot of co-immunoprecipitation (GFP-trap) after transient co-expression of interaction candidates in $N$. benthamiana. Total protein was stained with amido black. $\mathrm{Km}$, kinase mutated (K516A). C FRET-FLIM of AtLORE-Km-GFP and indicated candidates fused to an mCherry epitope tag, transiently co-expressed in $N$. benthamiana. Pooled data of five independent biological replicates. Data show mean with SD. n, number of analyzed cells. Statistics analyzed by one-way ANOVA with Tukey's multiple comparisons test, $\alpha=0.01$. Data not sharing the same letter are significantly different. D FRET-FLIM of AtLORE-Km-GFP with AtLORE-mCherry, transiently co-expressed in $\mathrm{N}$. benthamiana and treated with $5 \mu \mathrm{M} 3-\mathrm{OH}-\mathrm{C} 10: 0$ or $\mathrm{H}_{2} \mathrm{O}$ as control. FLIM images were acquired 10-20 minutes after treatment. Pooled data from two independent biological replicates. $n$, number of analyzed cells. Data show mean with SD. Differences between treatments were analyzed by two-way ANOVA with Sidak's multiple comparisons test, $\alpha=0.01$. 

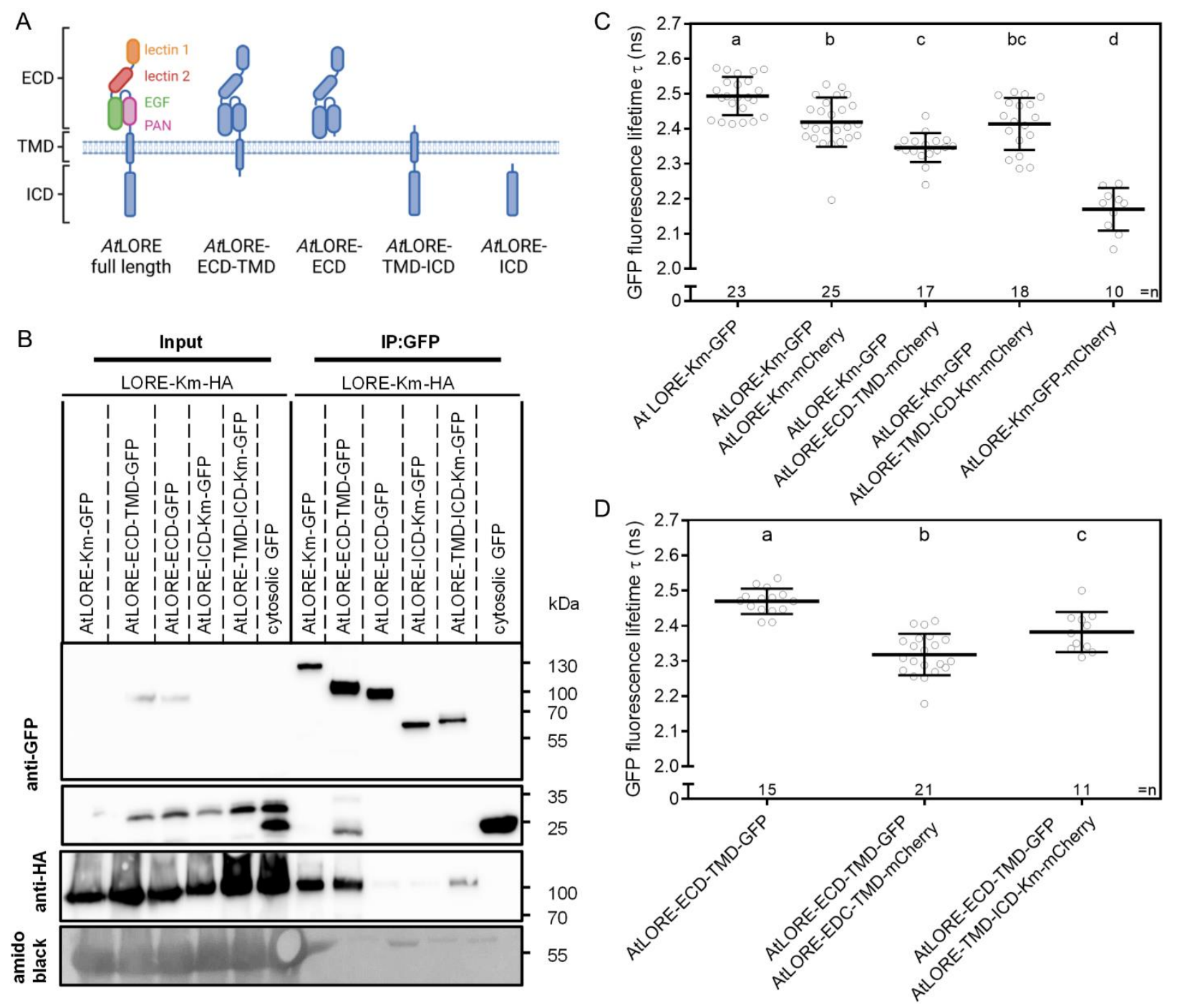

666
Figure 2 Homomerization of AtLORE is mediated by the extracellular and trans-membrane domain. A Scheme of AtLORE truncation variants; ECD, extracellular domain; TMD, transmembrane domain; ICD, intracellular domain. Created with BioRender.com. B Anti-GFP and anti-mCherry immunoblot of co-immunoprecipitation (GFP-trap) after transient co-expression of full length and truncated AtLORE-Km interaction candidates in $N$. benthamiana. Total protein is stained with amido black. C, D FRET-FLIM of AtLORE-Km-GFP full length or AtLORE-ECD-TMDGFP versus truncated AtLORE-mCherry variants transiently expressed in $N$. benthamiana. Pooled data from two independent biological replicates each. Data show mean with SD. $n$, number of analyzed cells. Statistics analyzed by one-way ANOVA with Tukey's multiple comparisons test, $\alpha=0.01$, data not sharing the same letter are significantly different. 

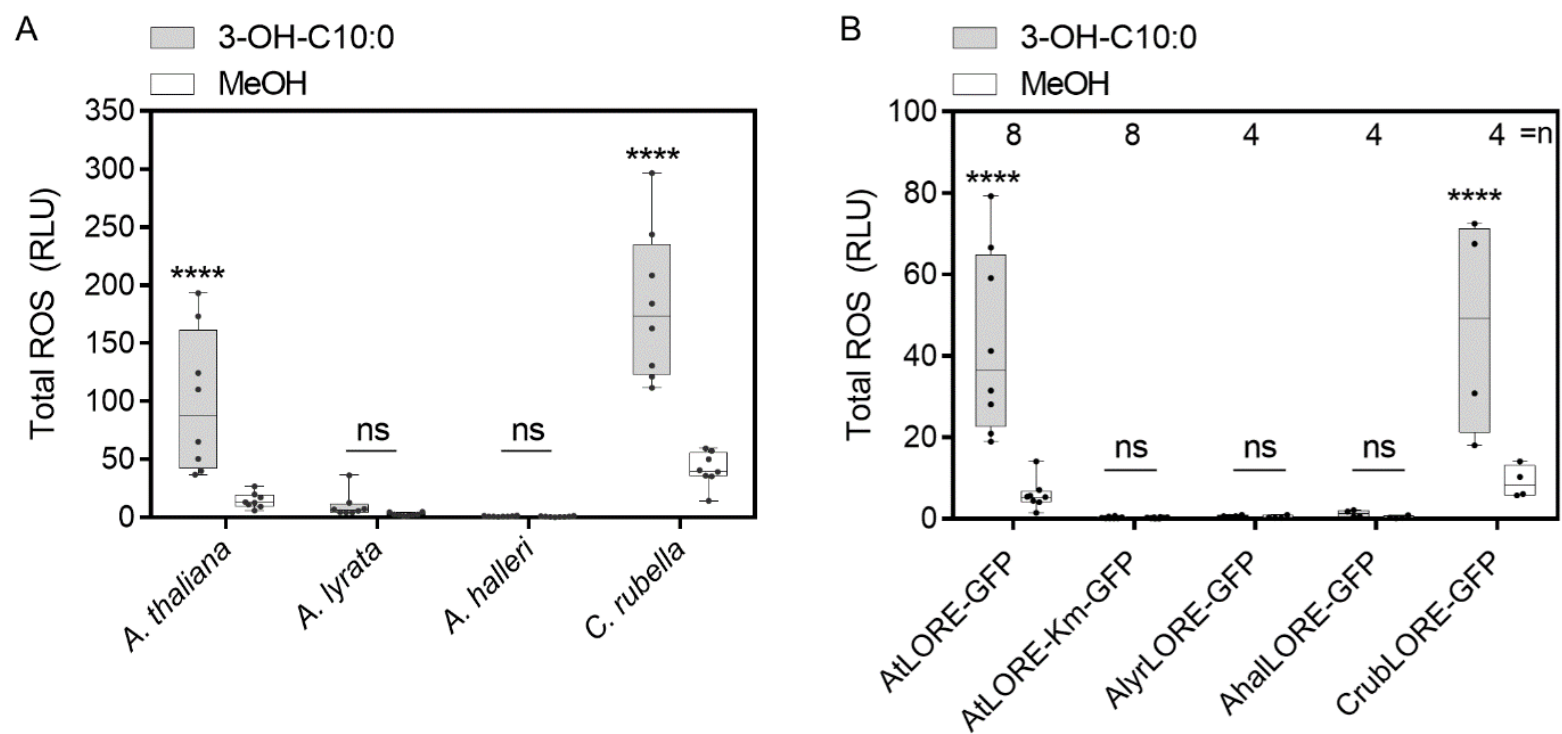

Figure 3 3-OH-C10:0 responsiveness of $A$. lyrata, $A$. halleri and $C$. rubella or $N$. benthamiana expressing putative LORE orthologues. A Total ROS accumulation of $A$. halleri, $C$. rubella, $A$. lyrata and $A$. thaliana leaf discs treated with $5 \mu \mathrm{M} 3-\mathrm{OH}-\mathrm{C} 10: 0$ or $\mathrm{MeOH}$ as control. Median with minimum to maximum of total ROS between 3-60 minutes after elicitation is shown; $n=8$ leaf discs. Significance between $\mathrm{MeOH}$ and 3-OH-C10:0 treatment tested with two-way ANOVA with Sidak's multiple comparisons test, $\alpha=0.01$. B Total ROS accumulation of $N$. benthamiana leaf discs transiently overexpressing AtLORE or putative LORE orthologues upon application of $1 \mu \mathrm{M}$ 3-OHC10:0 or MeOH as control. Boxplot displays minimum to maximum with median of total ROS between 3-45 minutes after treatment. $n$, number of leaf discs. Combined data of two independent datasets from one biological replicate are shown. For AtLORE and AtLORE-Km data of both datasets were pooled. Statistics was analyzed by two-way ANOVA with Sidak's multiple comparisons test, $\alpha=0.01$. 


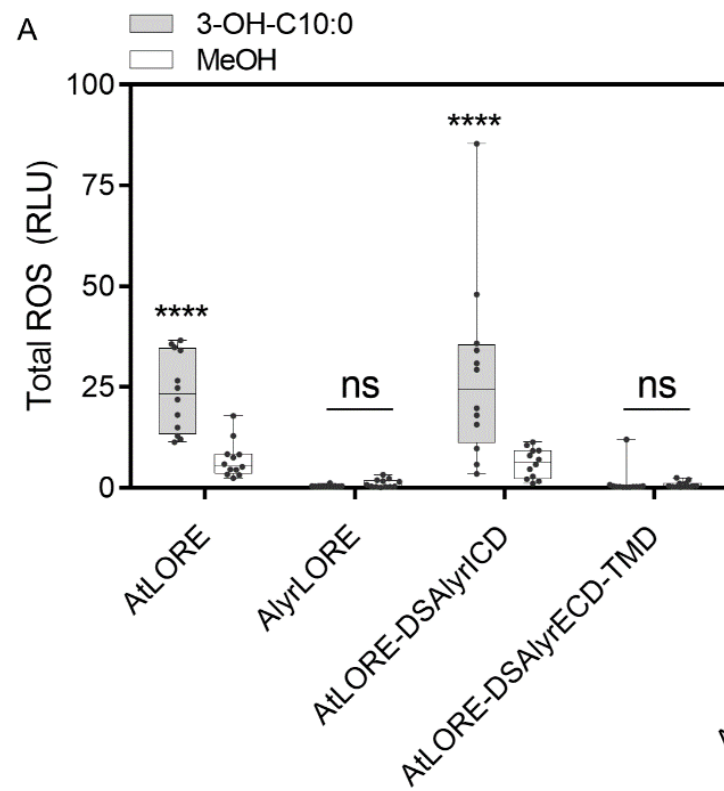

B

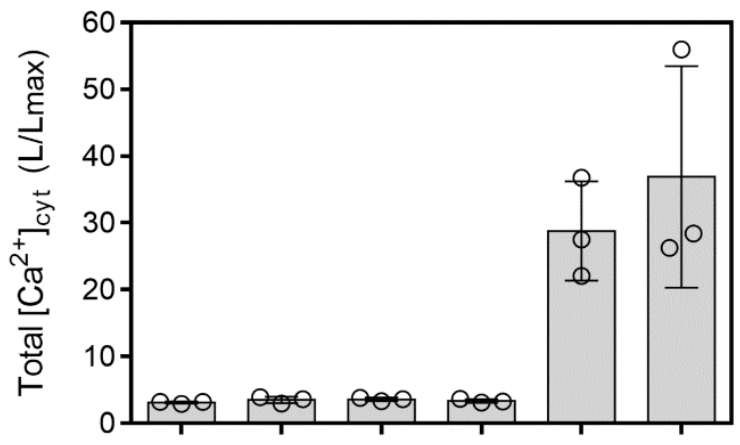

Figure 4 The ECD of AlyrLORE is causal for defective 3-OH-C10:0 perception, but ligand binding of all orthologues is functional. A Total ROS accumulation of AtLORE, AlyrLORE and chimera with either ECD-TMD or ICD domain swap (DS) elicited with $10 \mu \mathrm{M} 3-\mathrm{OH}-\mathrm{C} 10: 0$ or $\mathrm{MeOH}$. Median with minimum to maximum is shown; $n=12$ leaf discs. Statistics was analyzed by two-way ANOVA with Sidak's multiple comparisons test, $\alpha=0.01$. B 3-OH-C10:0 binding to LORE-ECDs was tested in a ligand depletion assay. Unbound 3-OH-C10:0 in filtrates was detected by cytosolic $\mathrm{Ca}^{2+}$ measurements of LORE-OE lines. Ligand binding is indicated by full depletion of a $\mathrm{Ca}^{2+}$ response. Data show mean and SD of total $\left[\mathrm{Ca}^{2+}\right]$ cyt from 3 to 30 minutes; $n=3$ seedlings. 
A

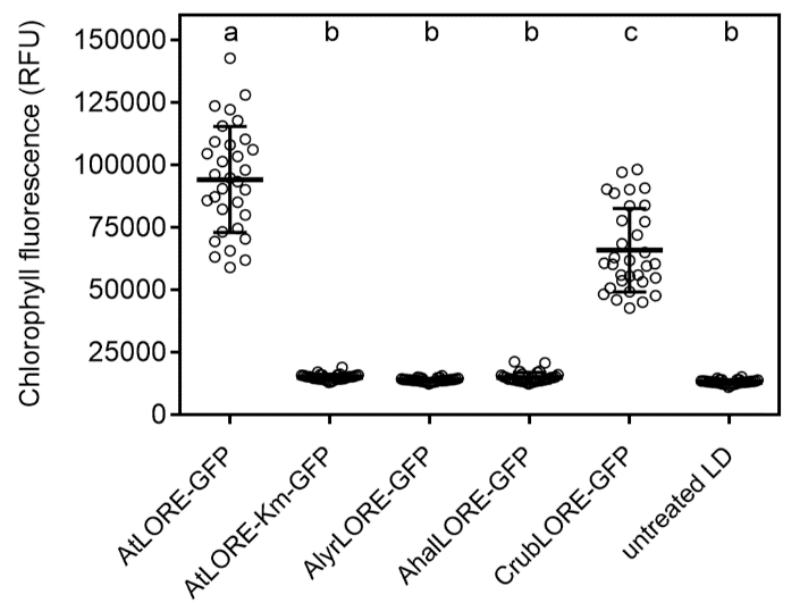

C

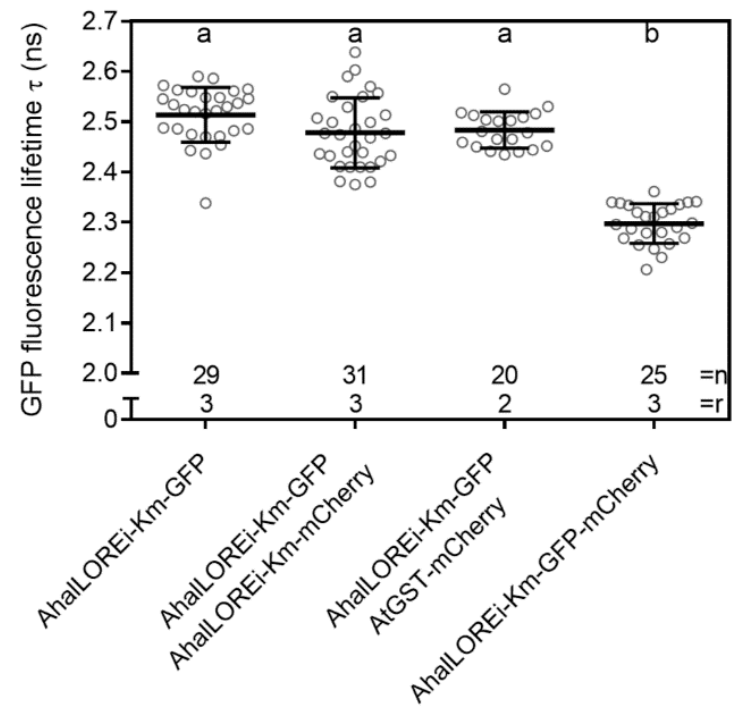

B

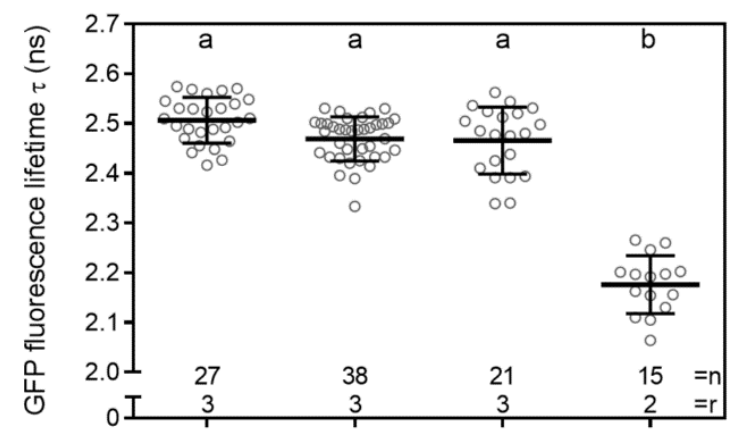

D
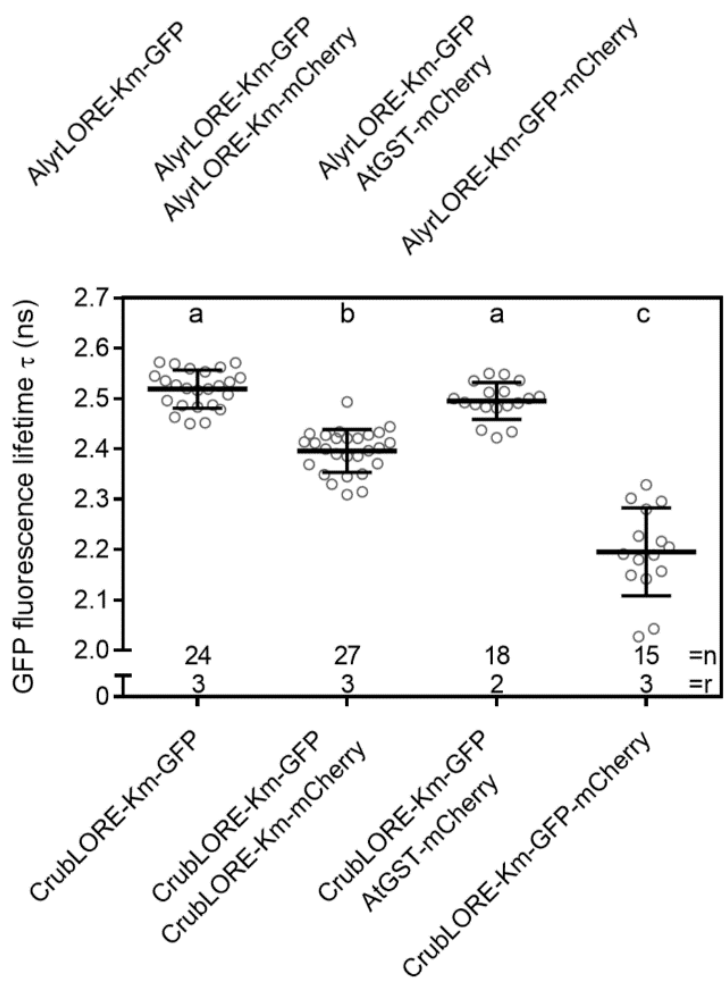

Figure 5 Putative LORE orthologues from 3-OH-C10:0 unresponsive species are impaired in homomerization. A Chlorophyll fluorescence measurement of $N$. benthamiana leaves transiently overexpressing AtLORE, AlyrLORE, AhalLORE and CrubLORE. Mean and SD of pooled data from two technical replicates are shown. $n=32$ leave discs. Statistics analyzed by oneway ANOVA with Tukey's multiple comparisons test, $\alpha=0.01$. Data not sharing data are significantly different. Experiment was repeated two times with similar outcome. B-D FRET-FLIM of putative LORE orthologous from A. lyrata (B) A. halleri (C) and C. rubella (D) transiently expressed in $N$. benthamiana. Km, kinase mutated; i, integrated LORE intron; $\mathrm{n}$, number of analyzed cells, $r$, number of biological replicates. Data show mean with SD. Statistics analyzed by one-way ANOVA with Tukey's multiple comparisons test, $\alpha=0.01$. Data not sharing the same letters are significantly different. 


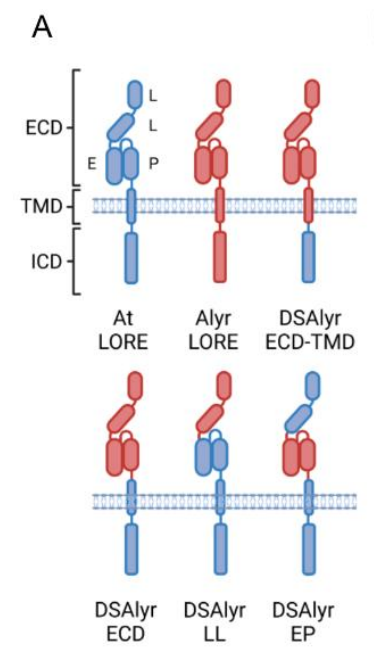

B
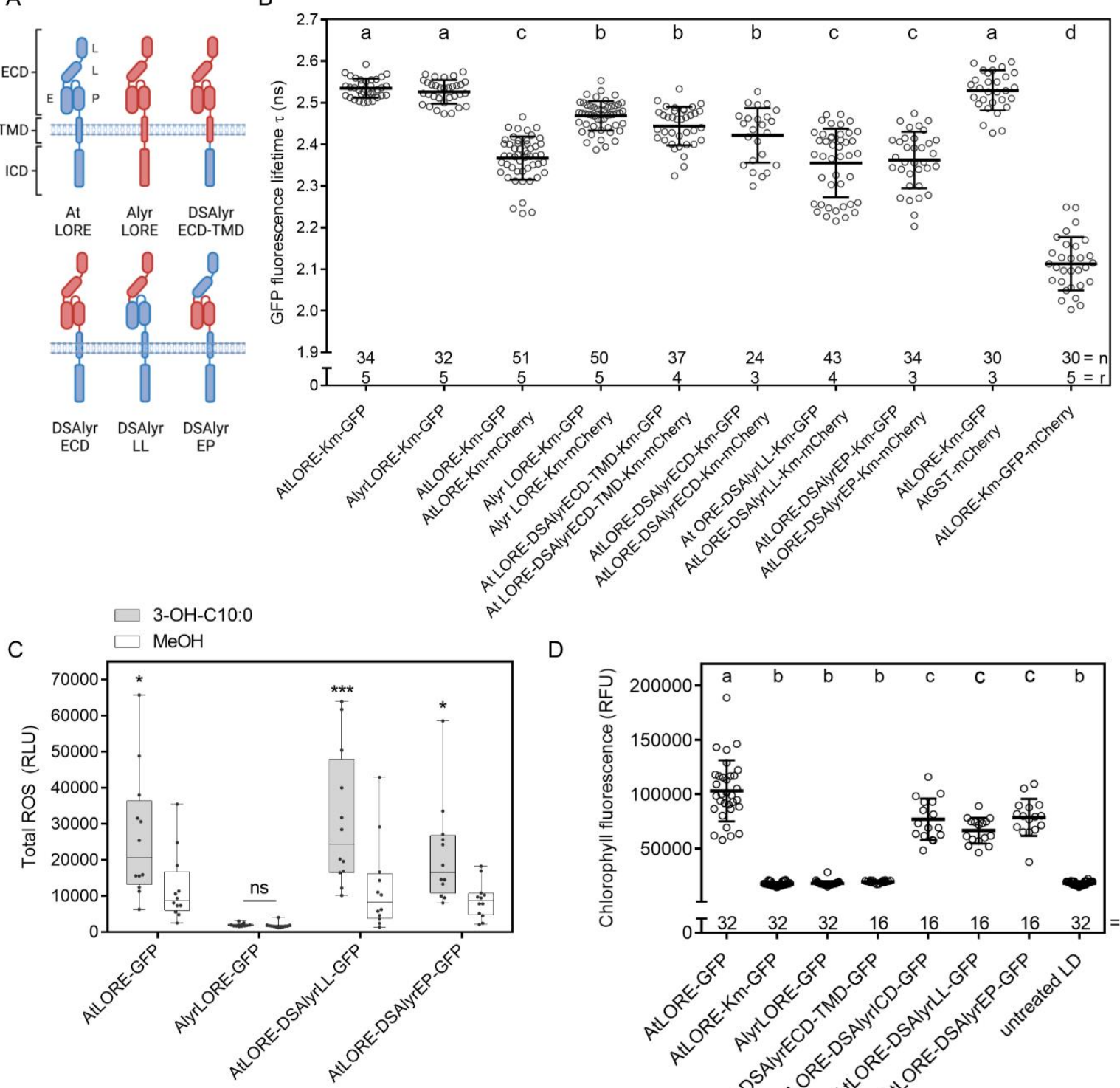

D

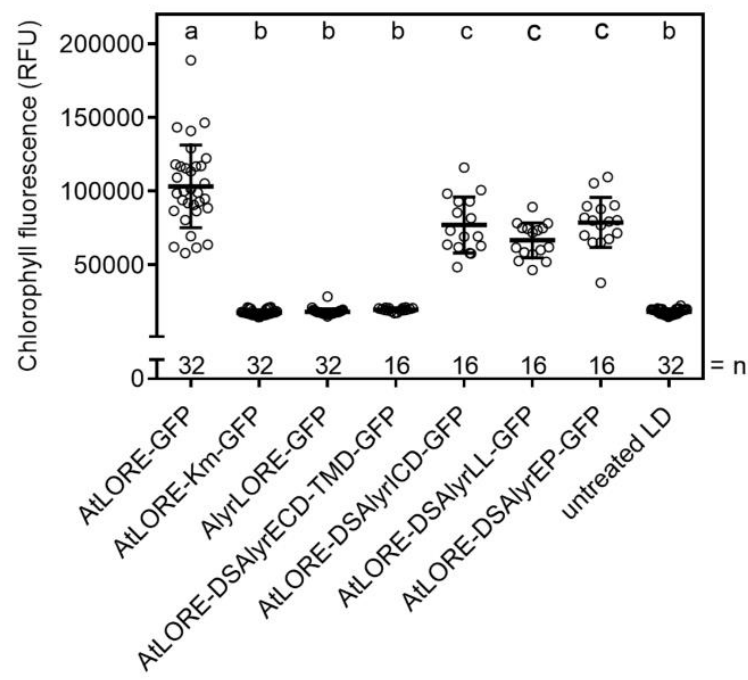

Figure 6 Mapping of dimerization region using receptor chimera of AtLORE and AlyrLORE. domain 1 and lectin domain 2; EP, EGF and PAN domain. Created with BioRender.com. B FRETFLIM of AtLORE-AlyrLORE domain swaps (DSAlyr) transiently expressed in $N$. benthamiana. Mean and SD of pooled data are shown; $r$, number of biological replicates; $n$, number of analyzed cells. Statistics analyzed by one-Way ANOVA with Tukey's multiple comparisons test, $\alpha=0.01$. Data not sharing the same letter are significantly different. $\mathbf{C}$ Gain of function ROS measurements of AtLORE-AlyrLORE chimera transiently expressed in $N$. benthamiana upon elicitation with $10 \mu \mathrm{M} 3-\mathrm{OH}-\mathrm{C} 10: 0$ or $\mathrm{MeOH}$ as control. Median with minimum to maximum is shown; $\mathrm{n}=12$ leaf discs. Statistics was analyzed by two-way ANOVA with Sidak's multiple comparisons test, $\alpha=0.01$. D Chlorophyll fluorescence measurements (in relative fluorescence units, RFU) in N. benthamiana upon transient expression of AtLORE-AlyrLORE chimera four days post transformation. Mean and $\mathrm{SD}$ of pooled data from two technical replicates of one biological replicate are shown. $n$, number of leaf discs. Statistics analyzed by one-way ANOVA with Tukey's multiple comparisons test, $\alpha=0.01$. Data not sharing the same letter are significantly different. 


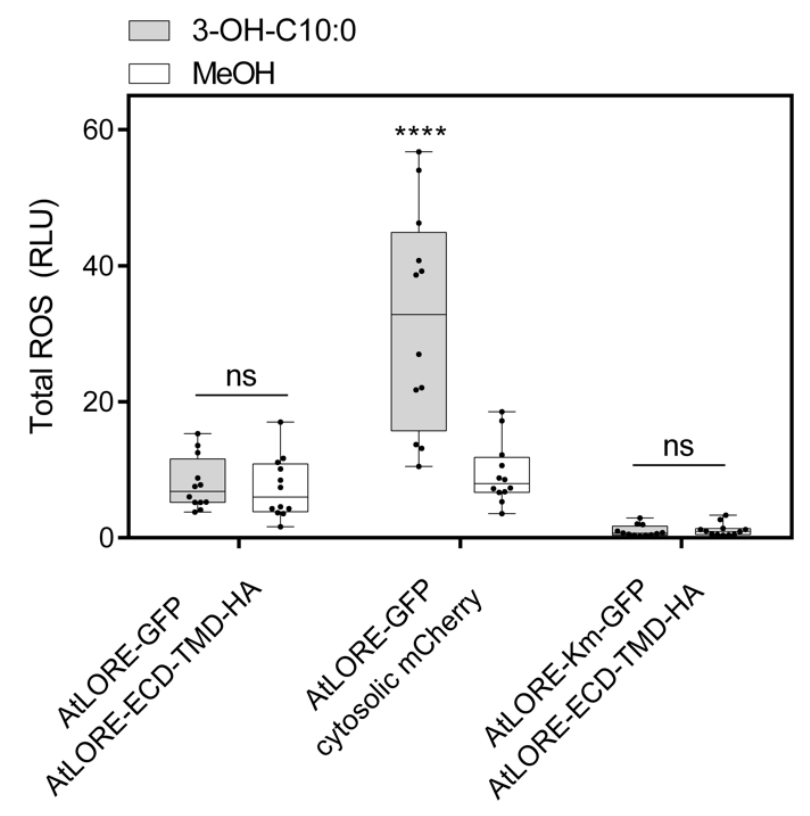

728 Figure 7 AtLORE homomerization is essential for downstream signaling. Dominant negative effects of co-expressed AtLORE-ECD-TMD-HA or cytosolic mCherry was analyzed by competitive ROS accumulation measurement in $N$. benthamiana transiently co-expressing indicated candidates upon elicitation with $5 \mu \mathrm{M} 3-\mathrm{OH}-\mathrm{C} 10: 0$ or $\mathrm{MeOH}$ as a control. Median with minimum to maximum of total ROS between 3 and 45 min from three technical replicates is shown; $n=12$ leaf discs. Differences between treatments was analyzed by two-way ANOVA and Sidak's multiple

734 comparisons test $(\alpha=0.01)$. 


\section{ACKNOWLEGEMENTS}

We acknowledge the Center for Advanced Light Microscopy (CALM) of imaging@TUM at the TUM School of Life Sciences for providing access to FLIM microscopes used in this study, Pascal Falter-Braun (Helmholtz center, Munich) and Marcel Quint (MLU Halle-Wittenberg) for providing plant material and Yvonne Stahl (HHU Düsseldorf) for providing FLIM vectors. Graphs and statistics were created by GraphPad Prism, microscopy images by OMERO and schemes by BioRender.com. We thank Ralph Hückelhoven and Martin Stegmann for critical discussion of data. Research in the Ranf lab is supported by the German Research Foundation (SFB924/TP-B10) and Emmy Noether program (RA2541/1).

\section{REFERENCES}

Allan, C., Burel, J.-M., Moore, J., Blackburn, C., Linkert, M., Loynton, S., MacDonald, D., Moore, W.J., Neves, C., Patterson, A., Porter, M., Tarkowska, A., Loranger, B., Avondo, J., Lagerstedt, I., Lianas, L., Leo, S., Hands, K., Hay, R.T., Patwardhan, A., Best, C., Kleywegt, G.J., Zanetti, G., and Swedlow, J.R. (2012). OMERO: flexible, model-driven data management for experimental biology. Nature Methods 9, 245-253.

Almagro Armenteros, J.J., Tsirigos, K.D., Sønderby, C.K., Petersen, T.N., Winther, O., Brunak, S., von Heijne, G., and Nielsen, H. (2019). SignalP 5.0 improves signal peptide predictions using deep neural networks. Nature biotechnology 37, 420-423.

Balint-Kurti, P. (2019). The plant hypersensitive response: concepts, control and consequences. Molecular Plant Pathology 20, 1163-1178.

Bauer, Z., Gómez-Gómez, L., Boller, T., and Felix, G. (2001). Sensitivity of Different Ecotypes and Mutants of $<$ em $>$ Arabidopsis thaliana $</$ em $>$ toward the Bacterial Elicitor Flagellin Correlates with the Presence of Receptor-binding Sites *. Journal of Biological Chemistry 276, 45669-45676.

Bi, G., Liebrand, T.W.H., Bye, R.R., Postma, J., Burgh, A.M., Robatzek, S., Xu, X., and Joosten, M.H.A.J. (2015). SOBIR1 requires the GxxxG dimerization motif in its transmembrane domain to form constitutive complexes with receptor-like proteins. Molecular Plant Pathology 17, 96-107.

Bleckmann, A., Weidtkamp-Peters, S., Seidel, C., and Simon, R. (2009). Stem Cell Signaling in Arabidopsis Requires CRN to Localize CLV2 to the Plasma Membrane. Plant physiology 152.

Bojar, D., Martinez, J., Santiago, J., Rybin, V., Bayliss, R., and Hothorn, M. (2014). Crystal structures of the phosphorylated BRI1 kinase domain and implications for brassinosteroid signal initiation. The Plant journal : for cell and molecular biology 78, 31-43.

Boller, T., and Felix, G. (2009). A Renaissance of Elicitors: Perception of Microbe-Associated Molecular Patterns and Danger Signals by Pattern-Recognition Receptors. Annual Review of Plant Biology 60, 379-406.

Boutrot, F., and Zipfel, C. (2017). Function, Discovery, and Exploitation of Plant Pattern Recognition Receptors for Broad-Spectrum Disease Resistance. Annual review of phytopathology 55, 257-286.

Burkart, R.C., and Stahl, Y. (2017). Dynamic complexity: plant receptor complexes at the plasma membrane. Curr Opin Plant Biol 40, 15-21.

Cao, Y., Liang, Y., Tanaka, K., Nguyen, C.T., Jedrzejczak, R.P., Joachimiak, A., and Stacey, G. (2014). The kinase LYK5 is a major chitin receptor in Arabidopsis and forms a chitininduced complex with related kinase CERK1. eLife 3, e03766.

Chen, L.J., Wuriyanghan, H., Zhang, Y.Q., Duan, K.X., Chen, H.W., Li, Q.T., Lu, X., He, S.J., Ma, B., Zhang, W.K., Lin, Q., Chen, S.Y., and Zhang, J.S. (2013). An S-domain 
receptor-like kinase, OsSIK2, confers abiotic stress tolerance and delays dark-induced leaf senescence in rice. Plant Physiol 163, 1752-1765.

Chen, X., Shang, J., Chen, D., Lei, C., Zou, Y., Zhai, W., Liu, G., Xu, J., Ling, Z., Cao, G., Ma, B., Wang, Y., Zhao, X., Li, S., and Zhu, L. (2006). A B-lectin receptor kinase gene conferring rice blast resistance. The Plant Journal 46, 794-804.

Cheng, X., Wu, Y., Guo, J., Du, B., Chen, R., Zhu, L., and He, G. (2013). A rice lectin receptorlike kinase that is involved in innate immune responses also contributes to seed germination. The Plant Journal 76, 687-698.

Chinchilla, D., Bauer, Z., Regenass, M., Boller, T., and Felix, G. (2006). The Arabidopsis receptor kinase FLS2 binds flg22 and determines the specificity of flagellin perception. The Plant cell 18, 465-476.

Chinchilla, D., Zipfel, C., Robatzek, S., Kemmerling, B., Nürnberger, T., Jones, J.D., Felix, G., and Boller, T. (2007). A flagellin-induced complex of the receptor FLS2 and BAK1 initiates plant defence. Nature 448, 497-500.

Consortium, T.U. (2020). UniProt: the universal protein knowledgebase in 2021. Nucleic acids research 49, D480-D489.

Couto, D., and Zipfel, C. (2016). Regulation of pattern recognition receptor signalling in plants. Nature reviews. Immunology 16, 537-552.

De Smet, I., Voß, U., Jürgens, G., and Beeckman, T. (2009). Receptor-like kinases shape the plant. Nature Cell Biology 11, 1166-1173.

Dievart, A., Gottin, C., Périn, C., Ranwez, V., and Chantret, N. (2020). Origin and Diversity of Plant Receptor-Like Kinases. Annual Review of Plant Biology 71, 131-156.

Engler, C., Gruetzner, R., Kandzia, R., and Marillonnet, S. (2009). Golden Gate Shuffling: A One-Pot DNA Shuffling Method Based on Type Ils Restriction Enzymes. PLOS ONE 4, e5553.

Fan, J., Bai, P., Ning, Y., Wang, J., Shi, X., Xiong, Y., Zhang, K., He, F., Zhang, C., Wang, R., Meng, X., Zhou, J., Wang, M., Shirsekar, G., Park, C.H., Bellizzi, M., Liu, W., Jeon, J.S., Xia, Y., Shan, L., and Wang, G.L. (2018). The Monocot-Specific Receptorlike Kinase SDS2 Controls Cell Death and Immunity in Rice. Cell host \& microbe 23, 498$510 . e 495$.

Fink, A., Sal-Man, N., Gerber, D., and Shai, Y. (2012). Transmembrane domains interactions within the membrane milieu: Principles, advances and challenges. Biochimica et Biophysica Acta (BBA) - Biomembranes 1818, 974-983.

Gilardoni, P.A., Hettenhausen, C., Baldwin, I.T., and Bonaventure, G. (2011). Nicotiana attenuata LECTIN RECEPTOR KINASE1 suppresses the insect-mediated inhibition of induced defense responses during Manduca sexta herbivory. The Plant cell 23, 35123532.

Giranton, J.L., Dumas, C., Cock, J.M., and Gaude, T. (2000). The integral membrane S-locus receptor kinase of Brassica has serine/threonine kinase activity in a membranous environment and spontaneously forms oligomers in planta. Proceedings of the National Academy of Sciences of the United States of America 97, 3759-3764.

Gomez-Gomez, L., and Boller, T. (2000). FLS2: an LRR receptor-like kinase involved in the perception of the bacterial elicitor flagellin in Arabidopsis. Molecular cell 5, 1003-1011.

Gong, B.-Q., Wang, F.-Z., and Li, J.-F. (2020). Hide-and-Seek: Chitin-Triggered Plant Immunity and Fungal Counterstrategies. Trends in plant science 25, 805-816.

Gou, X., and Li, J. (2020). Paired Receptor and Coreceptor Kinases Perceive Extracellular Signals to Control Plant Development. Plant physiology 182, 1667-1681.

Guo, X., Ye, J., Li, M., Lin, X., Lu, X., Liu, S., Li, H., and Zhang, C. (2018). LecRKIII.1 and LecRKIII.2 formed homodimers to play physiological functions in Arabidopsis thaliana. SDRP Journal of Plant Science 2, 1-8.

Herrmann, J.R., Panitz, J.C., Unterreitmeier, S., Fuchs, A., Frishman, D., and Langosch, D. (2009). Complex Patterns of Histidine, Hydroxylated Amino Acids and the GxxxG Motif 

Mediate High-affinity Transmembrane Domain Interactions. Journal of Molecular Biology 385, 912-923.

Hohmann, U., Lau, K., and Hothorn, M. (2017). The Structural Basis of Ligand Perception and Signal Activation by Receptor Kinases. Annu Rev Plant Biol 68, 109-137.

Ivanov, R., Fobis-Loisy, I., and Gaude, T. (2010). When no means no: guide to Brassicaceae self-incompatibility. Trends in plant science 15, 387-394.

Jamieson, P.A., Shan, L., and He, P. (2018). Plant cell surface molecular cypher: Receptor-like proteins and their roles in immunity and development. Plant Sci 274, 242-251.

Jany, E., Nelles, H., and Goring, D.R. (2019). Chapter One - The Molecular and Cellular Regulation of Brassicaceae Self-Incompatibility and Self-Pollen Rejection. In International Review of Cell and Molecular Biology, L. Galluzzi, ed (Academic Press), pp. 1-35.

Jinjun, Z., Peina, J., Fang, Z., Chongke, Z., Bo, B., Yaping, L., Haifeng, W., Fan, C., and Xianzhi, X. (2020). OsSRK1, an Atypical S-Receptor-Like Kinase Positively Regulates Leaf Width and Salt Tolerance in Rice. Rice Science 27, 133-142.

Kanzaki, H., Saitoh, H., Takahashi, Y., Berberich, T., Ito, A., Kamoun, S., and Terauchi, R. (2008). NbLRK1, a lectin-like receptor kinase protein of Nicotiana benthamiana, interacts with Phytophthora infestans INF1 elicitin and mediates INF1-induced cell death. Planta 228, 977-987.

Katoh, K., Rozewicki, J., and Yamada, K.D. (2019). MAFFT online service: multiple sequence alignment, interactive sequence choice and visualization. Briefings in Bioinformatics 20, 1160-1166.

Katzen, F. (2007). Gateway $((\mathrm{R}))$ recombinational cloning: a biological operating system. Expert opinion on drug discovery 2, 571-589.

Kim, H.S., Jung, M.S., Lee, S.M., Kim, K.E., Byun, H., Choi, M.S., Park, H.C., Cho, M.J., and Chung, W.S. (2009a). An S-locus receptor-like kinase plays a role as a negative regulator in plant defense responses. Biochem Biophys Res Commun 381, 424-428.

Kim, H.S., Jung, M.S., Lee, K., Kim, K.E., Yoo, J.H., Kim, M.C., Kim, D.H., Cho, M.J., and Chung, W.S. (2009b). An S-locus receptor-like kinase in plasma membrane interacts with calmodulin in Arabidopsis. FEBS Lett 583, 36-42.

Kutschera, A., Dawid, C., Gisch, N., Schmid, C., Raasch, L., Gerster, T., Schaffer, M., Smakowska-Luzan, E., Belkhadir, Y., Vlot, A.C., Chandler, C.E., Schellenberger, R., Schwudke, D., Ernst, R.K., Dorey, S., Huckelhoven, R., Hofmann, T., and Ranf, S. (2019). Bacterial medium-chain 3-hydroxy fatty acid metabolites trigger immunity in Arabidopsis plants. Science 364, 178-181.

Labbé, J., Muchero, W., Czarnecki, O., Wang, J., Wang, X., Bryan, A.C., Zheng, K., Yang, Y., Xie, M., Zhang, J., Wang, D., Meidl, P., Wang, H., Morrell-Falvey, J.L., Cope, K.R., Maia, L.G.S., Ané, J.-M., Mewalal, R., Jawdy, S.S., Gunter, L.E., Schackwitz, W., Martin, J., Le Tacon, F., Li, T., Zhang, Z., Ranjan, P., Lindquist, E., Yang, X., Jacobson, D.A., Tschaplinski, T.J., Barry, K., Schmutz, J., Chen, J.-G., and Tuskan, G.A. (2019). Mediation of plant-mycorrhizal interaction by a lectin receptor-like kinase. Nature Plants 5, 676-680.

Landeo Villanueva, S., Malvestiti, M.C., van leperen, W., Joosten, M.H.A.J., and van Kan, J.A.L. (2021). Red light imaging for programmed cell death visualization and quantification in plant-pathogen interactions. Molecular Plant Pathology 22, 361-372.

Langosch, D., and Arkin, I.T. (2009). Interaction and conformational dynamics of membranespanning protein helices. Protein Sci 18, 1343-1358.

Li, H., and Yang, W.-C. (2016). RLKs orchestrate the signaling in plant male-female interaction. Science China Life Sciences 59, 867-877.

Li, J.B., Sun, Y.D., Liu, H., Wang, Y.Y., Jia, Y.L., and Xu, M.H. (2015). Natural variation of rice blast resistance gene Pi-d2. Genetics and molecular research : GMR 14, 1235-1249. 
Liu, C., Dong, X., Xu, Y., Dong, Q., Wang, Y., Gai, Y., and Ji, X. (2021). Transcriptome and DNA Methylome Reveal Insights Into Phytoplasma Infection Responses in Mulberry (Morus multicaulis Perr.). Frontiers in Plant Science 12.

Liu, T., Liu, Z., Song, C., Hu, Y., Han, Z., She, J., Fan, F., Wang, J., Jin, C., Chang, J., Zhou, J.-M., and Chai, J. (2012). Chitin-Induced Dimerization Activates a Plant Immune Receptor. Science 336, 1160.

Luo, X., Wu, W., Liang, Y., Xu, N., Wang, Z., Zou, H., and Liu, J. (2020). Tyrosine phosphorylation of the lectin receptor-like kinase LORE regulates plant immunity. The EMBO journal n/a, e102856.

Ma, R., Han, Z., Hu, Z., Lin, G., Gong, X., Zhang, H., Nasrallah, J.B., and Chai, J. (2016a). Structural basis for specific self-incompatibility response in Brassica. Cell Res 26, 13201329.

Ma, X., Xu, G., He, P., and Shan, L. (2016b). SERKing Coreceptors for Receptors. Trends in plant science 21, 1017-1033.

Macho, A.P., Lozano-Durán, R., and Zipfel, C. (2015). Importance of tyrosine phosphorylation in receptor kinase complexes. Trends in plant science 20, 269-272.

Miya, A., Albert, P., Shinya, T., Desaki, Y., Ichimura, K., Shirasu, K., Narusaka, Y., Kawakami, N., Kaku, H., and Shibuya, N. (2007). CERK1, a LysM receptor kinase, is essential for chitin elicitor signaling in Arabidopsis. Proceedings of the National Academy of Sciences 104, 19613-19618.

Murase, K., Moriwaki, Y., Mori, T., Liu, X., Masaka, C., Takada, Y., Maesaki, R., Mishima, M., Fujii, S., Hirano, Y., Kawabe, Z., Nagata, K., Terada, T., Suzuki, G., Watanabe, M., Shimizu, K., Hakoshima, T., and Takayama, S. (2020). Mechanism of self/nonselfdiscrimination in Brassica self-incompatibility. Nature Communications 11, 4916.

Naithani, S., Chookajorn, T., Ripoll, D.R., and Nasrallah, J.B. (2007). Structural modules for receptor dimerization in the $S$-locus receptor kinase extracellular domain. Proceedings of the National Academy of Sciences 104, 12211-12216.

Nasrallah, J.B., and Nasrallah, M.E. (2014). S-locus receptor kinase signalling. Biochemical Society transactions $\mathbf{4 2 , 3 1 3 - 3 1 9 .}$

Navarro-Gochicoa, M.-T., Camut, S., Timmers, A.C.J., Niebel, A., Herve, C., Boutet, E., Bono, J.-J., Imberty, A., and Cullimore, J.V. (2003). Characterization of four lectin-like receptor kinases expressed in roots of Medicago truncatula. Structure, location, regulation of expression, and potential role in the symbiosis with Sinorhizobium meliloti. Plant physiology 133, 1893-1910.

Pan, J., Li, Z., Wang, Q., Yang, L., Yao, F., and Liu, W. (2020). An S-domain receptor-like kinase, OsESG1, regulates early crown root development and drought resistance in rice. Plant Sci 290, 110318.

Pietraszewska-Bogiel, A., Lefebvre, B., Koini, M.A., Klaus-Heisen, D., Takken, F.L.W., Geurts, R., Cullimore, J.V., and Gadella, T.W.J. (2013). Interaction of Medicago truncatula Lysin Motif Receptor-Like Kinases, NFP and LYK3, Produced in Nicotiana benthamiana Induces Defence-Like Responses. PLOS ONE 8, e65055.

Ranf, S., Gisch, N., Schaffer, M., Illig, T., Westphal, L., Knirel, Y.A., Sanchez-Carballo, P.M., Zahringer, U., Huckelhoven, R., Lee, J., and Scheel, D. (2015). A lectin S-domain receptor kinase mediates lipopolysaccharide sensing in Arabidopsis thaliana. Nat Immunol 16, 426-433.

Robatzek, S., and Wirthmueller, L. (2013). Mapping FLS2 function to structure: LRRs, kinase and its working bits. Protoplasma 250, 671-681.

Roux, M., Schwessinger, B., Albrecht, C., Chinchilla, D., Jones, A., Holton, N., Malinovsky, F.G., Tör, M., de Vries, S., and Zipfel, C. (2011). The Arabidopsis leucine-rich repeat receptor-like kinases BAK1/SERK3 and BKK1/SERK4 are required for innate immunity to hemibiotrophic and biotrophic pathogens. The Plant cell 23, 2440-2455. 
Schnepf, V., Vlot, A.C., Kugler, K., and Hückelhoven, R. (2018). Barley susceptibility factor RACB modulates transcript levels of signalling protein genes in compatible interaction with Blumeria graminis f.sp. hordei. Molecular plant pathology 19, 393-404.

Shimosato, H., Yokota, N., Shiba, H., Iwano, M., Entani, T., Che, F.-S., Watanabe, M., Isogai, A., and Takayama, S. (2007). Characterization of the SP11/SCR high-affinity binding site involved in self/nonself recognition in brassica self-incompatibility. The Plant cell 19, 107-117.

Shiu, S.H., and Bleecker, A.B. (2001). Plant receptor-like kinase gene family: diversity, function, and signaling. Science's STKE : signal transduction knowledge environment 2001 , re22.

Shiu, S.H., and Bleecker, A.B. (2003). Expansion of the receptor-like kinase/Pelle gene family and receptor-like proteins in Arabidopsis. Plant Physiol 132, 530-543.

Shiu, S.H., Karlowski, W.M., Pan, R., Tzeng, Y.H., Mayer, K.F., and Li, W.H. (2004). Comparative analysis of the receptor-like kinase family in Arabidopsis and rice. The Plant cell 16, 1220-1234.

Shu, L.-J., Schäffer, M., Eschrig, S., and Ranf, S. (2021). Low cost, medium throughput depletion-binding assay for screening S-domain-receptor ligand interactions using in planta protein expression. bioRxiv, 2021.2006.2016.448648.

Somssich, M., Ma, Q., Weidtkamp-Peters, S., Stahl, Y., Felekyan, S., Bleckmann, A., Seidel, C.A.M., and Simon, R. (2015). Real-time dynamics of peptide ligand-dependent receptor complex formation in planta. Science Signaling 8, ra76-ra76.

Stahl, Y., Grabowski, S., Bleckmann, A., Kühnemuth, R., Weidtkamp-Peters, S., Pinto, Karine G., Kirschner, Gwendolyn K., Schmid, Julia B., Wink, René H., Hülsewede, A., Felekyan, S., Seidel, Claus A.M., and Simon, R. (2013). Moderation of Arabidopsis Root Stemness by CLAVATA1 and ARABIDOPSIS CRINKLY4 Receptor Kinase Complexes. Current Biology 23, 362-371.

Stokes, K.D., and Gururaj Rao, A. (2008). Dimerization properties of the transmembrane domains of Arabidopsis CRINKLY4 receptor-like kinase and homologs. Archives of biochemistry and biophysics 477, 219-226.

Sun, M., Qian, X., Chen, C., Cheng, S., Jia, B., Zhu, Y., and Sun, X. (2018). Ectopic Expression of GsSRK in Medicago sativa Reveals Its Involvement in Plant Architecture and Salt Stress Responses. Frontiers in Plant Science 9.

Sun, W., Cao, Y., Jansen Labby, K., Bittel, P., Boller, T., and Bent, A.F. (2012). Probing the Arabidopsis flagellin receptor: FLS2-FLS2 association and the contributions of specific domains to signaling function. The Plant cell 24, 1096-1113.

Sun, X.L., Yu, Q.Y., Tang, L.L., Ji, W., Bai, X., Cai, H., Liu, X.F., Ding, X.D., and Zhu, Y.M. (2013a). GsSRK, a G-type lectin S-receptor-like serine/threonine protein kinase, is a positive regulator of plant tolerance to salt stress. Journal of plant physiology $170,505-$ 515.

Sun, Y., Qiao, Z., Muchero, W., and Chen, J.-G. (2020). Lectin Receptor-Like Kinases: The Sensor and Mediator at the Plant Cell Surface. Frontiers in plant science 11, 596301596301.

Sun, Y., Li, L., Macho, A.P., Han, Z., Hu, Z., Zipfel, C., Zhou, J.-M., and Chai, J. (2013b). Structural Basis for flg22-Induced Activation of the Arabidopsis FLS2-BAK1 Immune Complex. Science 342, 624-628.

Takayama, S., Shimosato, H., Shiba, H., Funato, M., Che, F.-S., Watanabe, M., Iwano, M., and Isogai, A. (2001). Direct ligand-receptor complex interaction controls Brassica selfincompatibility. Nature 413, 534-538.

Teixeira, M.A., Rajewski, A., He, J., Castaneda, O.G., Litt, A., and Kaloshian, I. (2018). Classification and phylogenetic analyses of the Arabidopsis and tomato G-type lectin receptor kinases. BMC Genomics 19, 239. 
989

990

991

992

993

994

995

996

997

998

999

1000

1001

1002

1003

1004

1005

1006

1007

1008

1009

1010

1011

1012

1013

1014

1015

1016

1017

1018

1019

1020

1021

1022

1023

1024

1025

1026

1027

1028

1029

1030

1031

1032
Vaid, N., Pandey, P.K., and Tuteja, N. (2012). Genome-wide analysis of lectin receptor-like kinase family from Arabidopsis and rice. Plant molecular biology 80, 365-388.

Walter, M., Chaban, C., Schütze, K., Batistic, O., Weckermann, K., Näke, C., Blazevic, D., Grefen, C., Schumacher, K., Oecking, C., Harter, K., and Kudla, J. (2004). Visualization of protein interactions in living plant cells using bimolecular fluorescence complementation. The Plant Journal 40, 428-438.

Wan, W.-L., Fröhlich, K., Pruitt, R.N., Nürnberger, T., and Zhang, L. (2019). Plant cell surface immune receptor complex signaling. Current Opinion in Plant Biology 50, 18-28.

Wang, Y., Cordewener, J.H.G., America, A.H.P., Shan, W., Bouwmeester, K., and Govers, F. (2015). Arabidopsis Lectin Receptor Kinases LecRK-IX.1 and LecRK-IX.2 Are Functional Analogs in Regulating Phytophthora Resistance and Plant Cell Death. Molecular Plant-Microbe Interactions $\AA^{8}$ 28, 1032-1048.

Waterhouse, A.M., Procter, J.B., Martin, D.M., Clamp, M., and Barton, G.J. (2009). Jalview Version 2--a multiple sequence alignment editor and analysis workbench. Bioinformatics (Oxford, England) 25, 1189-1191.

Weber, E., Engler, C., Gruetzner, R., Werner, S., and Marillonnet, S. (2011). A Modular Cloning System for Standardized Assembly of Multigene Constructs. PLOS ONE 6, e16765.

Weidtkamp-Peters, S., and Stahl, Y. (2017). The Use of FRET/FLIM to Study Proteins Interacting with Plant Receptor Kinases. Methods in molecular biology (Clifton, N.J.) 1621, 163-175.

Westerfield, J.M., and Barrera, F.N. (2020). Membrane receptor activation mechanisms and transmembrane peptide tools to elucidate them. The Journal of biological chemistry 295, 1792-1814.

Xing, S., Li, M., and Liu, P. (2013). Evolution of S-domain receptor-like kinases in land plants and origination of S-locus receptor kinases in Brassicaceae. BMC Evolutionary Biology $13,69$.

Xing, S., Wallmeroth, N., Berendzen, K.W., and Grefen, C. (2016). Techniques for the Analysis of Protein-Protein Interactions in Vivo. Plant Physiology 171, 727-758.

Xue, D.-X., Li, C.-L., Xie, Z.-P., and Staehelin, C. (2019). LYK4 is a component of a tripartite chitin receptor complex in Arabidopsis thaliana. Journal of experimental botany 70, 55075516.

Yu, X., Feng, B., He, P., and Shan, L. (2017). From Chaos to Harmony: Responses and Signaling upon Microbial Pattern Recognition. Annual review of phytopathology 55, 109137.

Zhou, D., Godinez-Vidal, D., He, J., Teixeira, M., Guo, J., Wei, L., Van Norman, J.M., and Kaloshian, I. (2021). A G-lectin Receptor Kinase is a Negative Regulator of Arabidopsis Immunity Against Root-Knot Nematode <em>Meloidogyne incognita</em>. bioRxiv, 2021.2009.2007.459316.

Zou, X., Qin, Z., Zhang, C., Liu, B., Liu, J., Zhang, C., Lin, C., Li, H., and Zhao, T. (2015). Over-expression of an S-domain receptor-like kinase extracellular domain improves panicle architecture and grain yield in rice. Journal of experimental botany $66,7197-$ 7209. 